\title{
The $l$-loop banana amplitude from GKZ systems and relative Calabi-Yau periods
}

\author{
Albrecht Klemm, ${ }^{a, b}$ Christoph Nega ${ }^{a}$ and Reza Safari ${ }^{a}$ \\ ${ }^{a}$ Bethe Center for Theoretical Physics, \\ Universität Bonn, D-53115 Bonn, Germany \\ ${ }^{b}$ Hausdorff Center for Mathematics, \\ Universität Bonn, D-53115 Bonn, Germany \\ E-mail: aklemm@th.physik.uni-bonn.de, cnega@th.physik.uni-bonn.de, \\ rsafari@th.physik.uni-bonn.de
}

ABSTRACT: We use the GKZ description of periods and certain classes of relative periods on families of Barth-Nieto Calabi-Yau $(l-1)$-folds in order to solve the $l$-loop banana amplitudes with their general mass dependence. As examples we compute the mass dependencies of the banana amplitudes up to the three-loop case and check the results against the known results for special mass values.

KEYwords: Differential and Algebraic Geometry, Scattering Amplitudes

ArXiv EPrint: 1912.06201 


\section{Contents}

1 Introduction 1

$2 \quad l$-loop banana diagram in the toric approach $\quad 4$

2.1 l-loop banana diagram 5

2.2 Geometry associated to $l$-loop banana diagram 5

2.2.1 Calabi-Yau hypersurfaces in toric ambient spaces 6

$\begin{array}{lll}2.2 .2 & \text { Period integrals on } M \text { and maximal cut amplitude } & 10\end{array}$

2.2.3 GKZ systems and Picard Fuchs differential ideal 12

$\begin{array}{lll}2.2 .4 & \text { Geometrical and physical periods } & 15\end{array}$

2.3 The complete banana diagram and inhomogeneous differential equations 16

3 Examples 17

$\begin{array}{ll}3.1 & \text { Example 1: the bubble graph } \\ & 17\end{array}$

$\begin{array}{lll}3.2 & \text { Example 2: the sunset graph } & 19\end{array}$

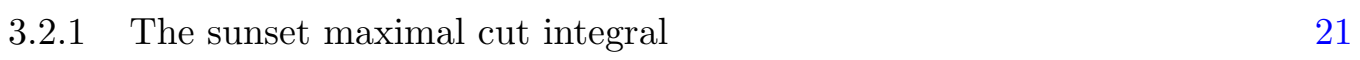

3.2.2 Extension to inhomogeneous differential operators 23

3.2.3 Comparison with the equal mass case and other known results 24

3.3 Example 3: the three-loop banana graph 25

$\begin{array}{lll}\text { 3.3.1 Maximal cut integral } & 27\end{array}$

$\begin{array}{lll}\text { 3.3.2 } & \text { Extension to inhomogeneous differential operators } & 29\end{array}$

3.3.3 The equal mass case and general properties of the ideal of differential operators 30

4 Conclusions and outlook $\quad 33$

A Differential operator ideal of the banana graph 35

\section{Introduction}

It was observed in [1] by Gel'fand, Kapranov and Zelevinskr (GKZ) that practically all integrals that arise in perturbative quantum field theory have the form of residuum integrals of rational functions defined in a toric variety $\mathbb{P}_{\Delta}$. We will call these the GKZ period integrals. In applications the more relevant statement is that in dimensional regularization say in $4-2 \epsilon$ dimensions the coefficients of the Laurent expansion of the Feynman integral in $\epsilon$ are such period integrals [2].

The simplest GKZ integrals are related to the Griffiths residuum form [3, 4] of geometric forms in the cohomology of varieties $M$ that are algebraically embedded in the toric 
varieties. The period integrals over closed cycles are solutions to a system of linear homogeneous differential operators called the Picard-Fuchs differential ideal (PFDI). The main result of GKZ is that the GKZ integrals are determined by a system of linear differential operators, w.r.t. to their parameters, defined in $[1,5,6]$ and called the GKZ system. This is of course only true up to linear combinations of the solutions, which reflects the choice of the homology class of the integration domain. This GKZ system can be thought as a generalization of the hypergeometric systems.

It is related in simple cases in which $M$ is a Calabi-Yau manifold to the PFDI as follows. After pulling out a moduli dependent multiplicative factor - the coefficient of the unique inner point in the Newton polyhedron - from the GKZ integrals, the geometric period integrals that solve the PFDI are among the solutions of the modified GKZ system ${ }^{1}[7,8]$. The application of $[7,8]$ shows that for many problems the PFDI is much easier obtained from the GKZ then from the Griffiths reduction method. The latter is a generic algorithm that produces differential relations between the periods by successive partial integrations, while the former uses simple symmetries of the integrand reflecting symmetries in the parameter space. More generally, the GKZ integrals can involve non necessary holomorphic forms that are integrated over chains in $M$ [9]. The latter are called relative periods and fulfill an inhomogeneous system of differential operators. It turns out that the solutions of GKZ systems can be related to periods as well as to a class of relative periods.

In this note we consider particular Feynman integrals that correspond to a class of $l$-loop Feynman diagrams in two space-time dimensions with two vertices of valence $l+1$, one invariant momentum $K^{2}$, and $l+1$ different masses $M_{i}$ for each propagator, known as Banana diagrams. These are depicted in figure 1. By the dimensional shift relations these integrals yield the leading terms in the dimensional regularization parameter $\epsilon$ in $4-2 \epsilon$ dimensions $[10,11]$. For these integrals ${ }^{2}$ given in $(2.1)$ the numerator of the rational function is trivial and the homogeneous differential system is related to the PFDI for the closed periods of the holomorphic $(l-1,0)$-form of a Calabi-Yau $(l-1)$-fold $M$. However, the integration domain of the Feynman integral has boundaries. It turns out that the solutions for the closed periods describe only the maximal cut integral of the Feynman amplitude. ${ }^{3}$ The latter is an important building block for the description of the physical amplitude, which however has to be supplemented by special solutions to the inhomogeneous equations, which correspond to the boundary contribution of the relative periods.

A fact of great importance for mirror symmetry is that Calabi-Yau manifolds are expected to have at least one point of maximal unipotent monodromy in their moduli space. For Calabi-Yau hypersurfaces and complete intersections in toric ambient spaces, the location of these points can in fact be calculated purely combinatorial from triangulations of the toric polyhedron. At such a point the local exponents for the solutions of the PFDI are

\footnotetext{
${ }^{1}$ As explained in $[7,8]$ the PFDI can be obtained from the modified GKZ system by factoring it from the latter.

${ }^{2}$ Also for other Feynman graphs the appearing integrals can be related to Calabi Yau integrals as pointed out in $[12-14]$.

${ }^{3}$ See also [15] for a connection between maximal cut Feynman integrals and solutions to corresponding differential equaions.
} 
completely degenerate. A consequence is that there is an unique analytic solution, while all other solutions are all logarithmic at this point. There is also an unique solution with the highest power of logarithms which equals the dimension $l-1$ of $M$. Moreover, the maximal cut integral corresponds to the unique holomorphic period and can be evaluated directly by a residuum integral over an $l$-dimensional torus in $\mathbb{P}_{\Delta}$. All logarithmic closed periods can be obtained by the Frobenius method. One of the technical insides of this paper is that also the inhomogeneous solutions can be constructed at this point from symmetries of the GKZ system and the explicit form of the GKZ integral and we show that this method ${ }^{4}$ is practical enough to calculate the full mass dependence for the three-loop amplitude and maybe beyond.

Only for the two-loop amplitude the dependence on all three parameters has been calculated so far $^{5}$ in [18]. The knowledge of the general mass dependence is not only important from a conceptual point but it is also required in the computation of higher loop corrections to certain processes studied at the Large Hadron Collider, as for example in Higgs production processes [19] the three-loop graph with two different masses is needed. It turned out that the two-loop integral is closely related to the period integral of the local mirror $M$ of the non-compact Calabi-Yau three-fold $W$ defined as total space of the anti-canonical line bundle over the degree three del Pezzo surface $S$, which is $\mathbb{P}^{2}$ blown up in three generic points. The masses are related in a simple way to the three new Kähler parameters in the blown up geometry and the toric polyhedron representing $S$ in figure 3. By local mirror symmetry the toric polyhedron is also the Newton polyhedron for the polynomial in the denominator of the GKZ integral, whose vanishing locus is a special family of elliptic curves $\mathcal{E}$, i.e. the Calabi-Yau one-fold. The period problem of the meromorphic differential of the third kind, whose non-vanishing residua correspond to the masses, on this elliptic curve has been solved universally for all toric del Pezzo surfaces in terms of modular forms [20]. It contains the information of the maximal cut integral.

The elliptic curve above will be replaced by a K3 for the three-loop case and a CalabiYau $(l-1)$-fold for the $l$-loop case. This banana diagram with general mass dependencies plays an important role in three-loop corrections to the $\rho$ parameter where the top and bottom quark masses are considered [21]. For the four-loop case the Calabi-Yau three-fold takes the form of a mass deformation of the one parameter family of Barth-Nieto quintics. Their form can be readily generalized to arbitrary dimensions as in equation (2.18) and will be called Barth-Nieto Calabi-Yau $(l-1)$-folds and describe the geometry of the $l$-loop graph (2.1) for equal masses ${ }^{6} \xi_{i}=M_{i} / \mu=1$ for all $i$, depending only on $t=K^{2} / \mu^{2}$. For the Barth-Nieto Calabi-Yau $(l-1)$-folds the $(l+1)$ 'th order Picard-Fuchs differential operator $\mathcal{D}_{t}^{(l+1)}$ is easily obtained. One evaluates the geometric integral (2.1) with $\xi_{i}=1$ over the $(l-1)$-torus $T^{l-1}$ instead of $\sigma_{l}$. The latter is readily performed as it is equivalent to an integral over an $T^{l}$ torus in the ambient space, which leads by a simple residue calculation to an explicit power series $\varpi(t)$. $\mathcal{D}_{t}^{(l+1)}$ can only have rational coefficients and

\footnotetext{
${ }^{4}$ See also $[16,17]$ for a different discussion of the GKZ system in the context of Feynman integrals with generic mass dependencies.

${ }^{5}$ In the two-loop case the diagram is also called sunset diagram.

${ }^{6}$ Due to an additional scaling freedom we can actually set in the equal mass case all masses to unity.
} 
demanding that it annihilates $\varpi(t)$ fixes it uniquely [22]. More efficiently is the method proposed in [23] using a decomposition of the integral in terms of Bessel functions. ${ }^{7}$

The full set of solutions to the Picard-Fuchs differential ideal and many aspects of their monodromies and analytic continuations have been intensively studied using the GKZ system in the context of mirror symmetry for period integrals of the holomorphic $(n, 0)$-form for Calabi-Yau n-folds. For compact Calabi-Yau three-folds realized as hypersurfaces in toric varities this was done in $[7,8,24]$ and for complete intersections in toric varieties in [25-27] Higher dimensional Calabi-Yau spaces have been studied in [28-32]. An introduction and overview into these subjects can be found in [33].

Our paper is structured as follows: in section 2.1 we introduce the $l$-loop banana graphs and explain their geometric interpretation. To these Feynman diagrams we associate $(l-1)$ dimensional Calabi-Yau hypersurfaces. Their definition and useful properties are discussed in section 2.2 and 2.2.1. In sections 2.2.2 to 2.2.4 we introduce the notion of periods and relative periods on Calabi-Yau hypersurfaces and explain the GKZ method. Moreover, we explain the restriction on the physical subslice. The extension of the GKZ system to relative periods is developed in 2.3. This is the main part of our approach of computing l-loop banana amplitudes. In section 3 we calculate with our approach three examples, namely the one-, two- and three-loop banana graph. Finally, we make our conclusions in section 4 .

Note added in draft: while we were in the process of finishing this draft an interesting paper [34] has been published about evaluating Feynman integrals and GKZ method which seems to have some overlap with our work.

\section{2 l-loop banana diagram in the toric approach}

We give the $l$-loop banana diagrams a geometric interpretation enabling us to use toric geometry to evaluate them. This geometric interpretation originates from the graph polynomial representation of a Feynman diagram which is obtained after Feynman parametrization and evaluation of many Gaussian integrals. ${ }^{8}$ For the banana type diagrams in two dimensions the exponent of the first Symanzik polynomial vanishes and the exponent of the second Symanzik polynomial is one. This simplifies the form of these integrals a lot and offers us a geometric interpretation familiar from string theory calculations. We regard the denominator of the integrand as a Newton polynomial which defines a Calabi-Yau hypersurface. The corresponding banana diagram integral is identified with a relative period of this Calabi-Yau hypersurface. Through the GKZ system of differential equations we construct a basis of periods on the Calabi-Yau variety at the maximal unipotent monodromy point. Extending the GKZ system to inhomogeneous differential operators we can write down a complete set of functions parametrizing the full banana amplitude.

\footnotetext{
${ }^{7}$ They are given up to $l=5$ together with a computer program that calculates them quickly for higher $l[23]$.

${ }^{8}$ For a review of the graph theoretical representation of Feynman diagrams we refer to [35].
} 


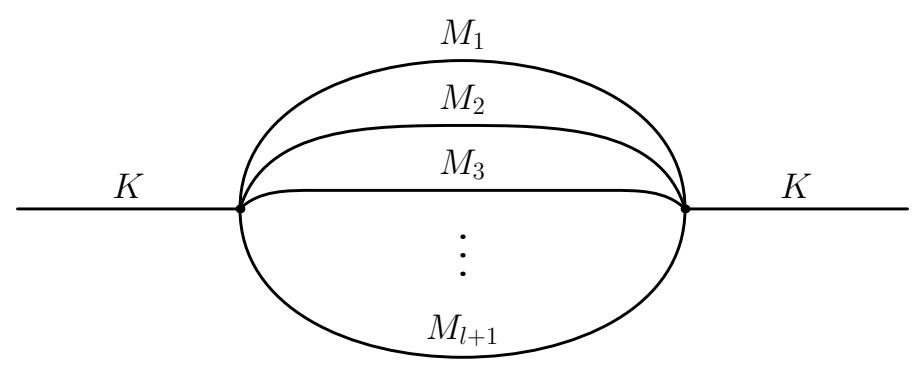

Figure 1. The l-loop banana diagram

\section{1 l-loop banana diagram}

The Feynman integral related to a l-loop banana diagram of a 2d QFT with the corresponding interactions drawn in figure 1 is given in Feynman parametrization as

$$
\mathcal{F}_{\sigma_{l}}\left(t, \xi_{i}\right)=\int_{\sigma_{l}} \frac{\mu_{l}}{P_{l}\left(t, \xi_{i} ; x\right)}=\int_{\sigma_{l}} \frac{\mu_{l}}{\left(t-\left(\sum_{i=1}^{l+1} \xi_{i}^{2} x_{i}\right)\left(\sum_{i=1}^{l+1} x_{i}^{-1}\right)\right) \prod_{i=1}^{l+1} x_{i}} .
$$

Here $x_{i}$ are the homogeneous coordinates of $\mathbb{P}^{l}$ and the $l$ real dimensional integration domain $\sigma_{l}$ is defined as

$$
\sigma_{l}=\left\{\left(x_{1}: \ldots: x_{l+1}\right) \in \mathbb{P}^{l} \mid x_{i} \in \mathbb{R} \text { with } x_{i} \geq 0, \forall i\right\} .
$$

The holomorphic $l$ measure $\mu_{l}$ is

$$
\mu_{l}=\sum_{k=1}^{l+1}(-1)^{k+1} x_{k} \mathrm{~d} x_{1} \wedge \ldots \wedge \widehat{\mathrm{d} x_{k}} \wedge \ldots \wedge \mathrm{d} x_{l+1} .
$$

The parameters or moduli in (2.1), $t$ and $\xi$, are dimensionless: $t=\frac{K^{2}}{\mu^{2}}$ and $\xi=\frac{M_{i}}{\mu}$ for $i=1, \ldots l+1$, where $K$ is the external momentum, $M_{i}$ are the $l+1$ masses and $\mu$ is an infrared scale.

The key observation discussed more in the next subsection is that (2.1) is the GKZ period integral for a Calabi-Yau hypersurface in a toric ambient space.

\subsection{Geometry associated to l-loop banana diagram}

The zero locus of the denominator of the integral defines a singular family of $(l-1)$-fold Calabi-Yau hypersurfaces $M_{s}$ as

$$
M_{s}=\left\{P_{l}\left(t, \xi_{i} ; x\right)=0 \mid\left(x_{1}: \ldots: x_{l+1}\right) \in \mathbb{P}^{l}\right\} .
$$

Due to standard arguments, see e.g. [36], $M_{s}$ is a complex Kähler manifold with trivial canonical class $K=0$, hence a Calabi-Yau space. The first fact follows by the definition of $M_{s}$ as hypersurface in projective space $\mathbb{P}^{l}$ and the second as for a homogeneous polynomial $P_{l}$ of degree $\operatorname{deg}(P)$ in $\mathbb{P}^{l}$ the canonical class is given in terms of the hyperplane class $H$ 
of $\mathbb{P}^{l}$ as $[36]-K=c_{1}\left(T M_{s}\right)=[(l+1)-\operatorname{deg}(P)] H$ and $\operatorname{deg}(P)=(l+1)$. Note that given the scaling of (2.3) this degree makes the integrand of (2.1) well defined under the $\mathbb{C}^{*}$ scaling of the homogenous coordinates defining $\mathbb{P}^{l}$. Embedded in $\mathbb{P}^{l}$ the hypersurface is a singular Calabi-Yau space. Due to the Batyrev construction there is a canonical resolution of these singularities to define a smooth Calabi-Yau family, which we discuss next following [7, 24, 26, 33]. A Calabi-Yau manifold $M$ of complex dimension $n=l-1$ has two characteristic global differential forms. Since it is Kähler it has a Kähler $(1,1)$ form $\omega$ defining its Kähler - or symplectic structure deformations space. The triviality of the canonical class implies the existence of an unique holomorphic $(n, 0)$-form that plays a crucial role in the description of the complex structure deformations space of $M$.

\subsubsection{Calabi-Yau hypersurfaces in toric ambient spaces}

First we define a Newton polynomial $P_{\Delta_{l}}$ as

$$
P_{l}\left(t, \xi_{i} ; x\right)=: P_{\Delta_{l}} \prod_{i}^{l+1} x_{i}
$$

The exponents of each monomial of $P_{\Delta_{l}}$, w.r.t. to the coordinates $x_{i}, i=1, \ldots, l+1$, define a point in a lattice $\mathbb{Z}^{l+1}$. The convex hull of all these points in the natural embedding of $\mathbb{Z}^{l+1} \subset \mathbb{R}^{l+1}$ defines an $l$-dimensional lattice polyhedron. The dimension is reduced due to the homogeneity of $P_{\Delta_{l}}$ and we denote the polyhedron ${ }^{9}$ that lies in the induced lattice $\mathbb{Z}^{l} \subset \mathbb{R}^{l}$ by $\Delta_{l}$.

More concretely, picking the canonical basis $e_{i}$ for $\Lambda=\mathbb{Z}^{l} \subset \mathbb{R}^{l}=\Lambda_{\mathbb{R}}$ the $l(l+1)$ vertices defined by (2.1) and (2.5) span the polytope $\Delta_{l},{ }^{10}$ i.e.

$$
\Delta_{l}=\operatorname{Conv}\left(\left\{ \pm e_{i}\right\}_{i=1}^{l} \cup\left\{ \pm\left(e_{i}-e_{j}\right)\right\}_{1 \leq i<j \leq l}\right) .
$$

Note that $\Delta_{l}$ contains beside these vertices no further integral point other then the origen $\nu_{0}=(0, \ldots, 0)$. Moreover, $\Delta_{l}$ is integral and reflexive, which implies that the dual polytope $\hat{\Delta}_{l} \subset \hat{\Lambda}_{\mathbb{R}}$

$$
\hat{\Delta}_{l}=\left\{y \in \hat{\Lambda}_{\mathbb{R}} \mid\langle y, x\rangle \geq-1, \forall x \in \Delta_{l}\right\}
$$

is also an integral lattice polyhedron. Note that $\widehat{\hat{\Delta}_{l}}=\Delta_{l}$ and concretely $\hat{\Delta}_{l}$ is given by

$$
\hat{\Delta}_{l}=\operatorname{Conv}\left(\bigcup_{k=1}^{l} \bigcup_{r=1}^{\left(\begin{array}{l}
l \\
k
\end{array}\right)} \sum_{i=1}^{l} I_{i}^{(k), r} \hat{e}_{i} \cup \bigcup_{k=1}^{l} \bigcup_{r=1}^{\left(\begin{array}{l}
l \\
k
\end{array}\right)} \sum_{i=1}^{l}\left(-I_{i}^{(k), r} \hat{e}_{i}\right)\right),
$$

where $\hat{e}_{i}$ is a basis of the lattice $\hat{\Lambda}_{\mathbb{R}}$ and the $I^{(k), r} r=1, \ldots,\left(\begin{array}{l}l \\ k\end{array}\right)$ are the sets of all distinct permutations of $k$ ones and $l-k$ zeros. Indeed the $2\left(2^{l}-1\right)$ points listed in (2.8) are all integral points of $\hat{\Delta}_{l}$ beside the origin. For the polytope $\Delta_{l}$ itself it means that it has $2\left(2^{l}-1\right)$ faces. From the structure of the vertices of $\Delta_{l}$ it can be proven that there is no

\footnotetext{
${ }^{9}$ One calls $P_{\Delta_{l}}$ the Newton polynomial of $\Delta_{l}$ and $\Delta_{l}$ the Newton polyhedron of $P_{\Delta_{l}}$.

${ }^{10}$ For $l=1,2,3$ these polytopes are depicted in figures 2,3 and 4 .
} 
integral point in the facets of the dual polytope. The combinatorics of all facets of $\hat{\Delta}$ are equal, in particular they all have $2^{l-1}$ vertices.

A central theorem in the toric mirror construction of Batyrev [24] says that a smooth resolution $M$ of $M_{s}$ with trivial canonical class is given by the constraint

$$
P_{\Delta_{l}}=\frac{P_{l}(a ; x)}{\prod_{i=0}^{p} x_{i}}=\sum_{\nu^{(i)} \in \Delta_{l}} a_{i} \prod_{\hat{\nu}^{(k)} \in \hat{\Delta}_{l}} x_{k}^{\left\langle\nu_{i}, \hat{\nu}_{k}\right\rangle}=0
$$

in the coordinate ring $x_{i}$ of $\mathbb{P}_{\hat{\Delta}_{l}}$, where $\nu_{i}, i=1, \ldots, p$ and $\hat{\nu}_{k}, i=1, \ldots, \hat{p}$ run over all integer points in $\Delta_{l}$ and $\hat{\Delta}_{l}$ respectively. ${ }^{11}$ Here $I\left(\Delta_{l}\right)$ is the number of lattice points in $\Delta_{l}$ and $p=I\left(\Delta_{l}\right)-1$. Analogous definitions apply for $\hat{\Delta}_{l}$. Note that (2.9) defines an embedding of the physical parameters $t$ and $\xi_{i}, i=1, \ldots, l+1$ into convenient but redundant complex structure variables $a_{i} \in \mathbb{C}, i=0, \ldots, l-1$. Both the physical as well as the $a_{i}$ parameters are only defined up to scale. Note that we are a little cavaliar with the notations: the coordinate rings $x_{i}, i=1, \ldots, l+1$ in the definition $(2.1)$ and the one $x_{i}, i=1, \ldots, \hat{p}$ in (2.9) are of course different. However, we can get the former by blowing down the latter. This is achieved by setting a suitable subset of $\hat{p}-(l+1)$ of the latter $x_{i}$ variables to one. Likewise given $P_{\Delta_{l}}$ in $x_{i}, i=1, \ldots, l+1$ as in $(2.5)$ and all $\mathbb{C}^{*}$ action $(2.11)$ we can uniquely extend it to $\hat{p}$ variables $x_{i}$ by requiring that the extended polynomial (or strictly speaking the proper transform of $(2.5))$ is homogeneous, w.r.t. to all $\mathbb{C}^{*}$ rescalings in (2.11).

The space $\mathbb{P}_{\Delta_{l}}$ is a $l$-dimensional projective toric variety that can be associated to any reflexive lattice polyhedra $\Delta_{l}$ given a star triangulation ${ }^{12} \mathcal{T}$ of $\Delta_{l}$ as

$$
\mathbb{P}_{\Delta_{l}}=\frac{\mathbb{C}^{p}\left[x_{1}, \ldots, x_{p}\right] \backslash Z_{\mathcal{T}}}{\left(\mathbb{C}^{*}\right)^{p-l}}
$$

Here the $\mathbb{C}^{*}$ actions that are divided out are generated by

$$
x_{i} \mapsto x_{i}\left(\mu^{(k)}\right)^{l_{i}^{(k)}}, \quad \text { for } i=1, \ldots, p,
$$

where $\mu^{(k)} \in \mathbb{C}^{*}$ and the $l^{(k)}$ vectors span the $(p-l)$-dimensional space of all linear relations

$$
L=\left\{\left(l_{0}^{*}, l_{1}^{*}, \ldots, l_{p}^{*}\right) \in \mathbb{Z}^{p+1} \mid l_{0}^{*} \bar{\nu}_{0}+l_{1}^{*} \bar{\nu}_{1}+\ldots+l_{p}^{*} \bar{\nu}_{p}=0\right\}
$$

among the points

$$
\mathcal{A}=\left\{\bar{\nu}_{0}, \bar{\nu}_{1}, \ldots, \bar{\nu}_{p} \mid \bar{\nu}_{i}=\left(1, \nu_{i}\right), \nu_{i} \in \Delta_{l} \cap \mathbb{Z}^{l}\right\}
$$

The triangulation ${ }^{13} \mathcal{T}$ determine the set of generators $l^{(k)}$ of $L$ and the Stanley-Reisner ideal $Z_{\mathcal{T}}$. The latter describes loci in $\mathbb{C}^{p}\left[x_{1}, \ldots, x_{p}\right]$, which have to be excluded so that the

\footnotetext{
${ }^{11} P_{\Delta_{l}}$ is a Laurent polynomial in which the minimal degree of the $x_{i}$ is -1 , while $P_{l}(a ; x)=0$ is a polynomial constraint, which also defines a smooth manifold in the coordinate ring .

${ }^{12}$ In a star triangulation all $l$-dimensional simplices of the triangulation covering the reflexive polyhedron share the inner point, as one of their vertices.

${ }^{13} \Delta_{l}$ defines canonically a fan $\Sigma_{\Delta_{l}}$ and the definition of a smooth $\mathbb{P}_{\Delta_{l}}$ may require to add integer points outside $\Delta_{l}$ and to triangulate the fan $\Sigma_{\Delta_{l}}$. Such cases are discussed in $[7,8]$.
} 
orbits of the $\mathbb{C}^{*}$ action (2.11) have a well defined dimension. Positive linear combinations of $l^{(k)}, k=1, \ldots, n$ span the Mori cone, which is not necessary simplicial if $n>p-l$. It is dual to the Kähler cone of $\mathbb{P}_{\Delta_{l}}$ and all cones corresponding to all triangulations $\mathcal{T}$ of $\Sigma_{\Delta_{l}}$ form the secondary fan, see [37] for a review how to calculate the $l^{(k)}$ vectors and the StanleyReisner ideal combinatorial from a triangulation $\mathcal{T}$. This combinatorics is implemented in the computer package SageMath [38], which calculates the possible triangulations $\mathcal{T}$ and from them the generators $l^{(k)}$ and the Stanley Reisner ideal $Z_{\mathcal{T}}$.

The Calabi-Yau $(l-1)$-fold family defined as section of the canonical bundle $P_{\hat{\Delta}_{l}}=0$ of $\mathbb{P}_{\Delta_{l}}$ is by Batryrev [24] conjectured to be the mirror manifold $W=X_{\hat{\Delta}_{l}}$ of the manifold $M$, i.e. $(M, W)$ form a mirror pair with dual properties. A main implication of this proposal is that the complex structure deformation space of $M$ denoted by $\mathcal{M}_{\mathrm{CS}}(M)$ is identified with the complexified Kähler or stringy Kähler moduli space $\mathcal{M}_{\mathrm{KCS}}(W)$

$$
\mathcal{M}_{\mathrm{KCS}}(W)=\mathcal{M}_{\mathrm{CS}}(M)
$$

and vice versa. Note that the real Kähler moduli space is parametrized by the Kähler parameters $t_{k}^{\mathbb{R}}=\int_{\mathcal{C}_{k}} \omega$, where $\omega \in H^{1,1}(M)$ and $\mathcal{C}_{k}$ span a basis of holomorphic curves in $H_{1,1}(M, \mathbb{Z})$. In string theory the complexification is due to the Neveu-Schwarz twoform field $b$ also in $H^{1,1}(M)$. The complex variables $t_{k}=\int_{\mathcal{C}_{k}} \omega+i b, k=1, \ldots, h_{1,1}(M)$ parametrize locally the complexified moduli space $\mathcal{M}_{\mathrm{KCS}}(W)$ of $W$.

We will next discuss the space $\mathcal{M}_{c s}(M)$ of complex structure deformations of $M$. This space is redundantly parametrized by the complex coefficients $a_{i}, i=0, \ldots, l\left(\Delta_{l}\right)-1$ in (2.9). The $a_{i}$ are identified by $l+1$ scaling relations on the coordinates of $\mathbb{P}_{\hat{\Delta}_{l}}$ and the automorphism of $\mathbb{P}_{\hat{\Delta}_{l}}$ that leaves $M$ invariant but acts on the parametrizations of the polynomial constraint $P(a ; x)$. The latter one parameter families of identifications of the deformation parameters are in an one-to-one correspondence to the points inside codimension one faces of $\Delta_{l}$. Let us denote by $\Theta_{k}^{j}$ all faces of codimension $k$ in $\Delta_{l}$ labeled by $j . I\left(\Theta_{k}^{j}\right)$ denotes the number of lattice points contained in $\Theta_{k}^{j}$, while $I^{\prime}\left(\Theta_{k}^{j}\right)$ denotes the number of lattice points that lie in the interior of $\Theta_{k}^{j}$. With this notation $M$ has $I\left(\Delta_{l}\right)-(l+1)-\sum_{j} I^{\prime}\left(\Theta_{1}^{j}\right)$ independent complex structure deformations. They correspond to elements in $H^{1}(M, T M)$ and are unobstructed on a Calabi-Yau manifold $M$. The cohomology group $H^{1}(M, T M)$ is related to the cohomology group $H^{l-2,1}(M)$ via the contraction with the unique holomorphic $(l-1,0)$-form.

Equation (2.14) implies that in particular the complex dimensions of these spaces have to match, i.e. $h_{1,1}\left(X_{\hat{\Delta}_{l}}\right)=h_{l-2,1}\left(X_{\Delta_{l}}\right)$ and $h_{1,1}\left(X_{\Delta_{l}}\right)=h_{l-2,1}\left(X_{\hat{\Delta}_{l}}\right)$. From theses facts it follows that the dimensions of these important cohomology groups are given by counting integral points in the polytops ${ }^{14}$

$$
\begin{aligned}
h_{1,1}\left(X_{\Delta_{l}}\right) & \left.=I\left(\hat{\Delta}_{l}\right)-(l+1)-\sum_{j} I^{\prime}\left(\hat{\Theta}_{1}^{j}\right)+\sum_{j} I^{\prime}\left(\hat{\Theta}_{2}^{i}\right) I^{\prime}\left(\Theta_{l-2}^{i}\right)\right)=2^{l+1}-l-2 \\
h_{l-2,1}\left(X_{\Delta_{l}}\right) & \left.=I\left(\Delta_{l}\right)-(l+1)-\sum_{i} I^{\prime}\left(\Theta_{1}^{i}\right)+\sum_{i} I^{\prime}\left(\Theta_{2}^{i}\right) I^{\prime}\left(\hat{\Theta}_{l-2}^{i}\right)\right)=l^{2} .
\end{aligned}
$$

\footnotetext{
${ }^{14}$ The last terms after the first equal sign in the formulas in each line of (2.15) correspond to Kähler or complex structure deformations, which are frozen by the toric realization of the manifolds, respectively. Likewise the third terms are absent in our case. The last equality holds only for the polyhedra given in (2.6) and (2.8).
} 
For $l=3$ the Calabi-Yau manifold $M$ will be a nine-parameter family of polarized $K 3$ surfaces. In this case the transversal cycles in $h_{11}$ are counted $h_{11}^{T}=I\left(\Delta_{l}\right)-(l+1)=9$, i.e. in total one has eleven transcendental and eleven algebraic two-cycles, which are counted by $h_{11}^{A}=I\left(\hat{\Delta}_{l}\right)-(l+1)=11$. For $l=4$ the 16 -parameter family of Calabi-Yau three-fold has $h_{11}=26$ and $h_{21}=16$ and hence Euler number $\chi=40$. For $l=5$ the Calabi-Yau four-fold has $h_{31}=25, h_{11}=57, h_{21}=0$ and $\chi=540$. Using an index theorem [30] one gets $h_{22}=422$.

Since our polytope (2.6) has only $\sum_{i} I\left(\Theta_{l}^{i}\right)=l(l+1)$ corners and one inner points the manifold $M$ has $l^{2}$ complex structure deformations, which have to be eventually mapped to our physical parameters $t$ and $\xi_{i}$. Since the latter are equivalent up to scaling by $\mu$ we have $l+1$ independent physical parameters. Therefore, the map to the physical parameter space has a huge kernel for high $l$ and special effort has to be made to specify the relevant physical subspace of $\mathcal{M}_{\text {phys }}(M) \subset \mathcal{M}_{c s}(M)$ as described concretely in the example sections $3.1,3.2$ and 3.3 .

Actual properties of the smooth canonical resolution of $M_{s}$, in particular its Kähler cone, depend on the choice of the star triangulation $\hat{\mathcal{T}}$ of $\hat{\Delta}_{l}$. However, these detailed properties of the Kähler moduli space $\mathcal{M}_{\mathrm{KS}}(M)$ of $M$ do not affect the complex moduli space $\mathcal{M}_{\mathrm{CS}}(M)$ and the integral (2.1) over closed cycles, like $\mathcal{F}_{T^{l}}$, the integral over the $T^{l}$ torus. This maximal cut integral depends only on the complex structure parameters. The blow up coordinate ring allows however a useful description of the boundary contribution to $\mathcal{F}_{\sigma_{l}}$, see [39]. Moreover, the identification (2.14) turns out to be very useful to introduce suitable coordinates on $\mathcal{M}_{\mathrm{CS}}(M)$ to obtain solutions for the integral (2.1). Different star triangulations $\mathcal{T}$ of the polyhedra $\Delta_{l}$ correspond to different Kähler cones of the ambient space $\mathbb{P}_{\Delta_{l}}$ of $W$ and correspond eventually ${ }^{15}$ to different Kähler cones of $W$. Each choice of the Kähler cone of $W$, defines by mirror symmetry and the identification (2.14) canonical so called Batyrev coordinates $z_{i}, i=1, \ldots, h_{l-2,1}(M)=h_{1,1}(W)$ on $\mathcal{M}_{\mathrm{CS}}(M)$, at whose origin $z_{i}=0$ for all $i$ there is a point of maximal unipotent monodromy in $\mathcal{M}_{\mathrm{CS}}(M)$. The coordinates $z_{i}$ are ratios of the coefficients $a_{i}$ of $P_{\Delta_{l}}$ given for each triangulation by

$$
z_{k}=\left(-a_{0}\right)^{l_{0}^{(k)}} \prod_{i} a_{i}^{l_{i}^{(k)}}, \quad k=1, \ldots, p-l .
$$

The definition of the $z_{k}$ eliminates the scaling relation. Since in our case we have no codimension one points, i.e. no automorphism of $\mathbb{P}_{\hat{\Delta}}$ leaving the hypersurface invariant and further identifying the $a_{i}$ deformations, the $z_{k}$ are actual coordinates on $\mathcal{M}_{\mathrm{CS}}(M)$. In other simple cases one can restrict in the definition of $L(2.12)$ to linear relations of points, which are not in codimensions one, the general case is discussed in $[7,8]$. In the moduli space of $\mathcal{M}_{\mathrm{CS}}(M)$ as parametrized by the independent $a_{i}$, the $z_{k}$ are blow up coordinates resolving singular loci in discriminant components of the hypersurface $P_{\Delta_{l}}=0$ in $\mathcal{M}_{\mathrm{CS}}(M)$, so that these become in the resolved model of the complex moduli space $\widehat{\mathcal{M}_{\mathrm{CS}}}(M)$ intersection points of normal crossing divisors $D_{i}=\left\{z_{i}=0\right\}, i=1, \ldots, h_{l-2,1}(M)$.

Of particular significance in the geometric toric construction of the differentials on $M$ is the coefficient $a_{0}$ of the monomial $\prod_{i}^{l+1} x_{i}$ in $P(t, \xi ; x)$ corresponding to the inner point

\footnotetext{
${ }^{15}$ If all curves that bound the Kähler cone of $\mathbb{P}_{\Delta_{l}}$ descend to the hypersurface $W$.
} 
in $\Delta_{l}$, which is given in the physical parameters by

$$
u:=a_{0}=t-\sum_{i=1}^{l+1} \xi_{i}^{2} .
$$

The families that are just parametrized by $u$ with the coefficients of all other points set to one, i.e. in particular $\xi^{2}=1$ for all $i=1, \ldots, l+1$ is particularly symmetric. For $l=4$, i.e. Calabi-Yau three-folds, the family is known as the Barth-Nieto quintic. The form of this family is conveniently given by a complete intersection in $\mathbb{P}^{l+1}$ that can be readily generalized to the ones

$$
\sum_{i=1}^{l+2} x_{i}=0 \quad \text { and } \quad \sum_{i=1}^{l+1} \frac{1}{x_{i}}+\frac{1}{u x_{l+2}}=0 .
$$

By solving for $x_{l+2}$ and homogenizing one gets $P_{\Delta_{l}}=0$ in the equal mass case parametrized by $u$ and for equal masses $\xi_{i}^{2}=1$ for all $i=1, \ldots, l+1$.

\subsubsection{Period integrals on $M$ and maximal cut amplitude}

For the discussion of the period integrals, which are very close to the integral of interest (2.1), we start with a residue definition of the holomorphic $(n, 0)$-form $\Omega$ of the CalabiYau manifold $M$ of complex dimension $n=l-1$ defined as hypersurface in a toric ambient space

$$
\Omega=\oint_{\gamma} \frac{a_{0} \mu_{l}}{P_{l}(a ; x)},
$$

where $\gamma$ encircles the locus $P_{l}=0$ in the toric ambient space and $\mu_{l}$ was defined in (2.3). Given a basis $\Gamma_{i}$ of the cycles in the middle dimensional homology $H_{n}(M, \mathbb{Z})$ we can define closed string period integrals

$$
\Pi(\Gamma)=\int_{\Gamma} \Omega
$$

The closed string periods are directly relevant as one of them describes the maximal cut integral. Moreover, by the local Torelli theorem $h^{n-1,1}$ of them can serve as projective coordinates of $\mathcal{M}_{\mathrm{CS}}$ and by Griffiths transversality the periods fulfill differential relations for odd $n>1$, algebraic relations for $n=2$ and algebraic as well as differential relations for even $n>2$.

At the point of maximal unipotent monodromy that is specified as the origin of the Batyrev coordinates $z_{i}, i=1, \ldots h$ from (2.16), which are simply defined by the Mori cone $l^{(k)}$ vectors of the mirror $W$, the Picard-Fuchs differential ideal is maximally degenerate. This point is a point of maximal unipotent monodromy or short MUM-point. As a consequence that near the MUM-point there is exactly one holomorphic period, and for $k=1, \ldots, n$ there are $h_{h o r}^{n-k, k}$ periods whose leading multi-degree in $\log \left(z_{i}\right), i=1, \ldots, h^{n-1,1}$ is of order $k$. For Calabi-Yau $n$-folds with $n>2$ the full cohomology groups $H^{n, 0}, H^{n-1,1}$ are horizontal. By complex conjugation this holds also for $H^{1, n-1}$ and $H^{0, n}$. In particular, for Calabi-Yau three-folds the whole middle cohomology is horizontal. Beside this general structure an additional bonus in the case of Calabi-Yau spaces given by hypersurfaces in 
toric ambient spaces is that there is a $n$-cycle with the topology of a $n$-torus $T^{n} \in H_{n}(M, \mathbb{Z})$ which yields that holomorphic period $\varpi:=\Pi\left(T^{n}\right)$ explicitly. With the definitions (2.19) and (2.20) this integral yields an $(n+1)$-times iterated residue integral over an $T^{n+1}$ in the ambient space, that can be readily evaluated in terms of the $l^{(k)}$ vectors as

$$
\varpi=\oint_{\left|x_{1}\right|=0} \frac{d x_{1}}{2 \pi i} \ldots \oint_{\left|x_{n+1}\right|=0} \frac{d x_{n+1}}{2 \pi i} \frac{a_{0}}{P_{l}(a ; x)}=\sum_{\{\underline{k}\}} \frac{\Gamma\left(-\sum_{\alpha=1}^{h} l_{0}^{(\alpha)} k_{\alpha}+1\right)}{\prod_{l=1}^{p} \Gamma\left(\sum_{\alpha=1}^{h} l_{l}^{(\alpha)} k_{\alpha}+1\right)} \prod_{\alpha=1}^{h} z_{\alpha}^{k_{\alpha}} .
$$

Here we use the coordinate ring $x$ as in $(2.1)$ and set $x_{n+2}=1$. In the tuple $\{\underline{k}\}=$ $\left\{k_{1}, \ldots, k_{h}\right\}$ each $k_{i}$ runs over non negative integers $k_{i} \in \mathbb{N}_{0}$ and $p$ is defined in (2.12). Note that by definition the sum of the integer entries in each $l^{(k)}$ is zero, therefore they have negative entries. For hypersurfaces and complete intersections the $l_{0}^{(k)}$ entry is nonpositive $l_{0}^{(k)} \leq 0$ for all $k$. However, for $i>0$ the $l_{i}^{(k)}$ can have either sign. Poles of the $\Gamma$-function at negative integers in the denominator make the summand vanishing. This effectively restricts the range of the $\left\{k_{1}, \ldots, k_{h}\right\}$ to a positive cone

$$
\sum_{\alpha=1}^{h} l_{j}^{(\alpha)} k_{\alpha} \geq 0
$$

Restricting to the physical slice, i.e. to $\underline{z}\left(t, \xi_{i}\right)$ of the $l$-loop graph, means to parametrize the $a_{i}, i=0, \ldots, h=l^{2}$ by the physical variables $t, \xi_{i}$. Due to the definition of (2.16) one can find a splitting of the set of indices $\left\{\alpha_{1}, \ldots, \alpha_{h}\right\}$ into $\left\{\alpha_{1}, \ldots, \alpha_{l+1}\right\}$ and $\left\{\alpha_{l+2}, \ldots, \alpha_{h}\right\}$ so that the variables $\left\{z_{\alpha_{l+2}}, \ldots, z_{\alpha_{h}}\right\}$ are either set to constant values or identified with the variables $z_{\alpha_{j}}(t, \xi), i=1, \ldots, l+1$. A key observation in the examples is that the range (2.22) is such that the contribution from the summation over the $k_{\alpha_{j}}, j=l+2, \ldots h$ to each monomial $\prod_{i=1}^{l+1} z_{\alpha_{i}}^{k_{i}}$ is finite. This implies in that (2.21) can also be given nonredundantly in $l+1$ physical parameters $z_{\alpha_{j}}(t, \xi), i=1, \ldots, l+1$ exactly to arbitrary order. The range (2.22) and (2.21) can also be calculated directly as follows: expanding in the integrand $a_{0} / P_{l}(x, a)=\left[1 / \prod_{i} x_{i}\right]\left[1 /\left(1-1 / a_{0}(\ldots)\right)\right]$ the second factor as a geometric series and noticing that only the constant terms of it contribute to the integral yields the result. Applying this to the $P_{l}$ in $(2.1)$ yields the all $(l=n+1)$-loop maximal cut integrals

$$
\mathcal{F}_{T^{l}}\left(t, \xi_{i}\right)=\frac{\varpi\left(\underline{z}\left(t, \xi_{i}\right)\right)}{t-\sum_{i=1}^{l+1} \xi_{i}^{2}}
$$

as an exact series expansion with finite radius of convergence for regions in the physical parameters in which $z_{k}\left(t, \xi_{i}\right)$ are all small.

In principal, one can analytically continue this to all regions in the physical parameter space. This task can greatly aided if one knows the Picard differential ideal that annihilates $\varpi$ and all other periods. The derivation of the latter will be discussed in the next section. It certainly helps if one knows all other periods near $z_{k}=0$. Because of the structure of the logarithmic solutions at the MUM-point these can by easily given by the Frobenius method. This is done by introducing $h$ auxiliary deformation parameters $\rho_{\alpha}$ in

$$
\varpi(\underline{z}, \underline{\rho})=\sum_{\{\underline{k}\}} c(\underline{k}, \underline{\rho}) \underline{z}^{\underline{k}+\underline{\rho}},
$$


where $\underline{\underline{k}}^{\underline{k}+\underline{\rho}}:=\prod_{\alpha=1}^{h} z_{\alpha}^{k_{\alpha}+\rho_{\alpha}}$ and

$$
c(\underline{k}, \underline{\rho})=\frac{\Gamma\left(-\sum_{\alpha=1}^{h} l_{0}^{(\alpha)}\left(k_{\alpha}+\rho_{\alpha}\right)+1\right)}{\prod_{l=1}^{p} \Gamma\left(\sum_{\alpha=1}^{h} l_{l}^{(\alpha)}\left(k_{\alpha}+\rho_{\alpha}+1\right)\right.} .
$$

With this definition $\varpi(\underline{z})=\left.\varpi(\underline{z}, \underline{\rho})\right|_{\underline{\rho}=0}$ the $h^{n-1,1}$ linear logarithmic solutions are given by

$$
\Pi\left(\Gamma_{\alpha}\right)=\left[\left.\left(1 /(2 \pi i) \partial_{\rho_{\alpha}} \varpi(\underline{z}, \underline{\rho})\right]\right|_{\underline{\rho}=0}=1 /(2 \pi i) \Pi\left(T^{n}\right) \log \left(z_{\alpha}\right)+\mathcal{O}(z) .\right.
$$

It can be shown that $\Gamma_{\alpha} \in H_{m}(M, \mathbb{Z})$. All other solutions corresponding to the rest of the cycles $\Gamma_{\beta} \in H_{n}(M, \mathbb{Z})$ are of order $2 \leq k \leq n$ in the logarithms and of the form

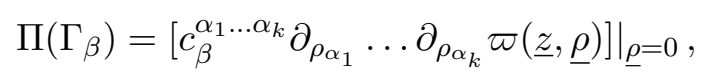

where the tensors $c_{\beta}^{\alpha_{1} \ldots \alpha_{k}}$ contain transcendental numbers fixed by the $\hat{\Gamma}$-class conjecture and classical intersection theory on $W$, see [33] for a review.

\subsubsection{GKZ systems and Picard Fuchs differential ideal}

Gel'fand, Kapranov and Zelevinskǐ [5] investigated integrals of the from

$$
\mathcal{F}_{\sigma}^{\mathrm{GKZ}}=\int_{\sigma} \prod_{i=1}^{r} P\left(x_{1}, \ldots, x_{k}\right)^{\alpha_{i}} x_{1}^{\beta_{1}} \cdots x_{k}^{\beta_{k}} \mathrm{~d} x_{1} \cdots \mathrm{d} x_{k},
$$

which can be specialized to (2.1), which is in turn similiar to (2.21), even though in (2.21) we took the integration domain to be a closed cycle $T^{n+1}$, while [5] just speek of cycles $\sigma$.

In (2.1) $\sigma$ is a closed cycle only for the maximal cut case which leads to (2.21), otherwise $\sigma$ is a chain. In this case the corresponding differential ideal, which is fulfilled by the integral (2.28) is inhomogeneous. The GKZ integrals can be viewed as systematic multivariable generalization of the Euler integral ${ }_{2} F_{1}(a, b, c ; z)=\sum_{n=0}^{\infty} \frac{(a)_{n}(b)_{n}}{n !(c)_{n}}=$ $\frac{\Gamma(c)}{\Gamma(b) \Gamma(b-c)} \int_{0}^{1} t^{(b-1)}(1-t)^{(b-c-1)}(1-z t)^{-a}$, which solves Gauss hypergeometric systems and $\varpi$ as a specially simple generalized multivariable hypergeometric series.

As mentioned at the end of the introduction to subsection (2.2) at least for integer exponents the requirement that these higher dimensional integrals are well defined under the scaling symmetries of the parameters, that appear in physical Feynman integrals, is equivalent to the vanishing of the first Chern class and hence these Feynman integrals with $r=1, \alpha_{1}=-1=-n_{1}$ are closely related to period integrals over the holomorphic $(n, 0)$ form in the cohomology group $H^{n, 0}$ of the Calabi-Yau manifolds $M$ defined as hypersurfaces in toric varieties $[7,8,40]$. The same argument relates integrals with $r>1$ and $\alpha_{i}=-1=$ $-n_{i}$ to complete intersection Calabi-Yau spaces [25-27].

More general integrals are related to the former by taking derivatives w.r.t. to the independent complex moduli parameters say $a$. In particular, such derivatives change the Hodge type of the integrand as follows. Let $F^{p}(M)=\bigoplus_{l \geq p} H^{l, n-l}(M)$ a Hodge filtration $H^{n}=F^{0} \supset F^{1} \supset \ldots \supset\left(F^{n}=H^{n, 0}\right) \supset F^{n+1}=0$, then $H^{p, q}(M)=F^{p}(M) \cap \overline{F^{q}(M)}$, and 
the $F^{p}(M)$ can be extended to holomorphic bundles $\mathcal{F}^{p}(M)$ over the complex family $M$ over $\mathcal{M}_{\mathrm{CS}}(M)$, with

$$
\partial_{a}^{k} \mathcal{F}^{n}(M) \in \mathcal{F}^{n-k}(M) .
$$

Since the bundles $\mathcal{F}^{p}(M)$ are of finite rank, there will be differential relations among finite derivatives w.r.t. to the moduli, which implies that the period integrals over closed cycles are annihilated by finite order linear differential operators $\mathcal{D}_{k}$, where the derivations are w.r.t. the moduli and the coefficients are rational functions in the moduli. In particular, one can specify a differential ideal, called the Picard-Fuchs differential ideal, $\mathcal{D}_{k}, k=1, \ldots, d$ that determines the periods as finite linear combination of its system of solutions.

One key tool to find the differential relations between these integrals is the Griffiths reduction method, which relies on the following partial integration formula, that is valid up to exact terms, i.e. holds under the integration over closed cycles [9]

$$
\sum_{k \neq j} \frac{n_{k}}{n_{j}-1} \frac{P_{j}}{P_{k}} \frac{Q \partial_{x_{i}} P_{k}}{\prod_{l=1}^{r} P_{l}^{n_{l}}} \mu=\frac{1}{n_{j}-1} \frac{P_{j} \partial_{x_{i}} Q}{\prod_{l=1}^{r} P_{l}^{n_{l}}} \mu-\frac{Q \partial_{x_{i}} P_{j}}{\prod_{l=1}^{r} P_{l}^{n_{l}}} \mu
$$

where $Q(x)$ are polynomials of the appropriate degree to ensure the scale invariances and $\mu$ is straightforward generalization of the measure (2.3). Such $Q(x)$ arise automatically, when partial derivative w.r.t. the moduli are taken. Using these equations and Gröber basis calculus one can reduce higher derivatives w.r.t. to the moduli to lower ones and find eventually the complete differential ideal. These relations between rational functions are also used in the literature not only to compute differential equations for Feynman integrals but also for finding so called master integrals. If these master integrals are known with the partial integration relations (2.30) the whole Feynman integral is evaluated. For a review on master integrals in Feynman graph computation we refer to [41].

However, this method is computationally very expensive in multi moduli cases. Therefore, we employ as far as possible a different derivation of differential relations which follow from scaling symmetries that follow from the combinatorics of the Newton polytope, known as GKZ differential system. For this purpose we define

$$
\hat{\Omega}=\oint_{\gamma} \frac{\mu_{l}}{P_{l}(a ; x)} \quad \text { and } \quad \hat{\Pi}_{\sigma}=\int_{\sigma} \frac{\mu_{l}}{P_{l}(a ; x)} .
$$

Now each linear relation among the points in the Newton polytope as expressed by the $l^{(k)}$-vectors, $k=1, \ldots, l^{2}$ yields a differential operator $\mathcal{D}_{l^{(k)}}$ in the redundant moduli $a$. Moreover, the infinitesimal invariance under the $\left(\mathbb{C}^{*}\right)^{n+2}$ scaling relations yields further differential operators $\mathcal{Z}_{j}, j=1, \ldots, n+2$. Together they constitute an resonant GKZ system $[1,6]$ :

$$
\begin{aligned}
\hat{\mathcal{D}}_{l^{(k)}} \hat{\Pi}_{\sigma} & =\left(\prod_{l_{i}^{(k)}>0}\left(\frac{\partial}{\partial a_{i}}\right)^{l_{i}^{(k)}}-\prod_{l_{i}^{(k)}<0}\left(\frac{\partial}{\partial a_{i}}\right)^{-l_{i}^{(k)}}\right) \hat{\Pi}_{\sigma}=0 \quad \text { and } \\
\mathcal{Z}_{j} \hat{\Pi}_{\sigma} & =\left(\sum_{i=0}^{p} \bar{\nu}_{i, j} \theta_{a_{i}}-\beta_{j}\right) \hat{\Pi}_{\sigma}=0
\end{aligned}
$$


with $\beta=(-1,0, \ldots, 0) \in \mathbb{R}^{n+2}$ for the hypersurface case and $\theta_{a}=a \partial_{a}$, in the form that applies to the integrals in Calabi-Yau hypersurfaces in toric varieties [7, 24], for which the integration domain $\sigma$ is also scale invariant. In this case we can use the relations $\mathcal{Z}_{j} \hat{\Pi}_{\sigma}=0$ to eliminate the $a_{i}$ in favour of the scale invariant $z_{i}$ defined in (2.16) using $a_{i} \partial_{a_{i}}=\sum_{k=1}^{l^{2}} l_{i}^{(k)} z_{k} \partial_{z_{k}}$ and by the commutation relation $\left[\theta_{a}, a^{r}\right]=r a^{r}$ applied previously to $a_{0}$ we obtain operators $\mathcal{D}_{l^{(k)}}(z)$ that annihilate $\Pi(\Gamma)$. As it turns out these operators do not determine the $\Pi(\Gamma)$ as they admit further solutions [7]. To obtain the actual PicardFuchs differential ideal one can factorize the $\mathcal{D}_{l^{(k)}}(z)$ and disregard trivial factors that allow for additional solutions which have the wrong asymptotic to be periods [7, 25]. In practice the most efficient way to get the Picard-Fuchs differential ideal is often to make an ansatz for additional minimal order differential operators that annihilate (2.21) and check that the total system of differential operators allows no additional solutions then the ones specified in $(2.26)$ and $(2.27)$.

One of our main results is that we give the general strategy to derive the Picard-Fuchs differential ideal in the physical parameters $z_{i}(t, \xi), i=1, \ldots, l+1$ and give it explicitly for one, two and three loops in equations (3.5), (3.25) and in (A.1)-(A.4) for the threeloop banana graph. These operators determine the maximal cut integral everywhere in the parameter space. By applying these operators to the geometrical chain integral

$$
\Pi_{\sigma_{l}}=\int_{\sigma_{l}} \frac{a_{0} \mu_{l}}{P_{l}(a ; x)}
$$

and integrating explicitly over the boundary of the chain we can find the inhomogeneous differential equations and the corresponding special solutions describing the full $l$-loop banana graphs explicitly up to three loops.

Let us end this section with some remarks on additional structures for the periods of Calabi-Yau $n$-folds, which are relevant to understand the differential ideal that determines the maximal cut integral better. For a given basis of transcendental $n$-cycles $\Gamma_{i} \in H_{n}(M, \mathbb{Z})$ one can find dual elements $\gamma_{j} \in H_{\text {hor }}^{n}(M, \mathbb{C})$ so that $\int_{\Gamma_{i}} \gamma^{j}=\delta_{i}^{j}$ and expand the holomorphic $(n, 0)$-form $\Omega=\sum_{i} \Pi\left(\Gamma_{i}\right) \gamma^{i}$. Let us define for each set $A$ of indices of order $r$ the order $r$ differential operator $\partial_{A}^{r}:=\partial_{z_{a_{1}}} \ldots \partial_{z_{a_{r}}}$. Then by (2.29) and consideration of type one gets the transversality conditions [42]

$$
\int_{M} \Omega \wedge \partial_{A}^{r} \Omega=\Pi\left(\Gamma_{i}\right) \Sigma^{i j} \partial_{A}^{r} \Pi\left(\Gamma_{j}\right)= \begin{cases}0 & \text { if } r<n \\ C_{A}(z) & \text { if } r=n,\end{cases}
$$

where $C_{A}$ are rational functions in the $z_{i}$, known as Yukawa couplings for $n=3$. The form $\Sigma^{i j}=\int_{M} \gamma^{i} \wedge \gamma^{j}$ is integer and symmetric for $n$ even and antisymmetric for $n$ odd. In the latter case one can chose a symplectic basis for the $\gamma^{i}$. For the K3 or more generally $n \geq 2$ and $n$ even it implies that the solutions to the Picard-Fuchs differential ideal fulfill nontrivial quadratic relations

$$
\Pi\left(\Gamma_{i}\right) \Sigma^{i j} \Pi\left(\Gamma_{j}\right)=0 \quad \text { and } \quad \Pi\left(\Gamma_{i}\right) \Sigma^{i j} \partial_{z_{k}} \Pi\left(\Gamma_{j}\right)=0, \quad \text { for all } k .
$$

We will discuss the consequences at the level of the differential operator more in section 3.3.3. For $n=3$ it implies special geometry, see [33] for a review. 


\subsubsection{Geometrical and physical periods}

The physical moduli space of the banana Feynman diagrams is parametrized by the $l+2$ parameters $\left(t, \xi_{1}, \xi_{2}, \ldots, \xi_{l+1}\right)$, where additionally one of these can be scaled away. As mentioned, compared to the moduli space parametrized by all Batyrev coordinates $z_{i}$ the physical moduli space is much smaller. In the following we explain how one can make a restriction onto the physical moduli space.

Besides this restriction there is another difficulty we have to mention. For the description of the large moduli space through the Batyrev coordinates $z_{i}$ it is crucial to have a minimal number of Mori cone generators. They are determined from the triangulation $\mathcal{T}$ of the polytope ${ }^{16} \Delta_{l}$. There are only finitely many fine and star-triangulations such that it is not directly clear that there exists a triangulation which yield $l^{2}$ Mori cone generators. Actually, for the sunset graph this is the case. In such a situation one starts with a triangulation yielding a non-minimal number of Mori cone generators. We claim that one can still take out $l^{2} l$-vectors describing the Feynman graph geometry appropriately. The choice of $l^{2}$ vectors is neither arbitrary nor unique but we can give some criteria ${ }^{17}$ for choosing them correctly. Different proper selections of $l$-vectors should at the end yield the same results for the Feynman graph.

First of all the $l^{2}$ vectors should be all linear independent over the real numbers. Secondly, we want $l+1 l$-vectors having a non-vanishing entry for the inner point which are important in the physical limit. Furthermore, we want that in the $i$-th components of all $l$-vectors there is at least a positive entry. This should be true for all components $i$ without the one for the inner point. From the last condition we hope that it guarantees that the structure of solutions is as we explained in section 2.2.2. This one can check by analyzing that the GKZ operators defined in (2.33) do indeed annihilate the Frobenius solutions with positive powers (2.24).

We think that these conditions give a strategy to take out the required $l^{2}$ mori cone generators. For the sunset graph we have to follow this strategy and we give the results in section 3.2. Although there exist fine and star-triangulations with nine $l$-vectors for the three-loop banana diagram, we nevertheless applied our criteria on a non-simplicial cone. Also in the three-loop case the criteria select a proper set of nine $l$-vectors yielding the same results as presented in section 3.3 computed from a triangulation with minimal number of mori cone generators.

Now the restriction onto the physical moduli space starts with using the inequalities (2.22) such that the holomorphic solution (2.21) is evaluated exactly in the physical relevant Batyrev coordinates. Having found this period on the physical slice we search for operators annihilating it such that the set of common solutions to these operators form a basis of the periods on the physical slice. This finally yields a basis of periods on the physical moduli space. It is quite hard to give a universal description of these operators. In general they form a differential operator ideal of linear, homogeneous differential oper-

\footnotetext{
${ }^{16}$ For $l=3$ one can easily get all $2^{6}$ star triangulations but for $l=4$ there is an extremely large number of different star triangulations, which we have estimated to be $6^{20}$. Listing all of them cannot be done by a desktop computer.

${ }^{17}$ We do not claim that these criteria are necessary or sufficient.
} 
ators and their explicit form as for example their degree depend on the representation of the ideal. For our discussion we write down an ansatz for a differential operator in terms of logarithmic derivatives of the remaining Batyrev coordinates. Thereby, we start with second order operators with polynomial coefficients which we make of smallest degree as possible. Typically, this ansatz yields a large number of possible operators from which we have to take a generating set of the differential ideal. From cohomology arguments we expect as many single logarithmic solutions as the number of interesting physical parameters, which strongly depends on the concrete banana diagram. Therefore, we take as many operators until their number of logarithmic solutions fits to the cohomological prediction. If the resulting solutions do still not satisfy all expectations, e.g. the number of higher logarithmic solutions, one has to extend the set of operators with higher degree ones until all expected solutions are determined. In this way one finds a generating set of operators for the differential ideal describing the physical periods. This part of our method depends strongly on the given form of the physical holomorphic period which is why we refer to our examples. We only remark that later it is crucial that the operators and the physical solutions are expressed in the remaining physical Batyrev coordinates.

\subsection{The complete banana diagram and inhomogeneous differential equations}

So far, we have found a complete differential ideal with solutions spanning a basis of the physical periods. Or said differently, these functions after dividing by the inner point describe the maximal cut integral $\mathcal{F}_{T^{l}}$. Now we extend our method to find the missing functions which complete the function space for the full banana Feynman diagram $\mathcal{F}_{\sigma_{l}}$. By function space we mean a set of functions which suitably combined yield the complete banana Feynman integral (2.1). It turns out that for the banana graphs there is only a single additional function we have to compute.

Basically, we extend the homogeneous differential ideal to a set of inhomogeneous differential operators such that its solutions describe the full Feynman graph. These inhomogeneities are found from the appropriate homogeneous operator by the following process: we let an operator directly act on the geometric differential, which is given as the integrand of (2.34), and perform then the integration over the domain $\sigma_{l}$. In this way we obtain for every homogeneous operator a corresponding inhomogeneous one.

For this task the original parametrization of the differential is changed to the Batyrev coordinates (2.16). This has a major advantage in the following. After applying the operators on the differential we can integrate over the simplex $\sigma_{l}$. In contrast to a period integral the integration range of the complete Feynman graph is not closed and such we get non zero after integration. Unfortunately, these integrals can not be carried out analytically with generic parameters. But they can be performed easily numerically. The advantage of including the inner point and using the Batyrev coordinates is now that the numerical results can simply be guessed. We claim that for the $l$-loop banana integrals they are only given as linear combinations of logarithms in the Batyrev coordinates. In our calculated examples given in section 3 we could always guess the inhomogeneities yielding a full set of inhomogeneous operators. 
In the literature there are already some methods known for computing relative periods in a way that homogeneous differential equations describing usual periods are extended to inhomogeneous ones. For examples in [43] a method for general toric varieties is explained how to extend the GKZ method to relative periods. The key point for this method is the $l$-vector description of the variety and its relative cohomology. The $l$-loop banana diagrams are not entirely described through $l$-vectors and therefore this method can not be applied. Moreover, there is the Dwork-Griffith reduction to obtain the homogeneous differential equations which then can analogously be extended to inhomogeneous ones as in our method [18]. Although Dwork-Griffith reduction can in principle be applied in any situation as explained before, for computational reasons only the sunset graph can explicitly be done. Compared with known methods our strategy uses the structure of the l-loop banana diagrams more efficiently and produces results also for high loop orders.

Having found the inhomogeneous operators its solutions are given by the solutions of the homogeneous operators together with a single special solution of the inhomogeneous system. A special solution is found by an ansatz which has a similar logarithmic structure as the homogeneous solutions. Only the power of the highest appearing logarithm is increased by one compared to the other solutions. This closes the set of functions describing the $l$-loop banana Feynman graph.

Our method gives a relatively small set of functions necessary to compute the banana graphs. For example, with numerical computations the correct linear combination of these functions evaluating to the Feynman graph can be fixed. We exemplify this on the sunset graph in section 3.2.3. Moreover, a detailed analysis of the analytic structure of these functions based on the inhomogeneous differential equations can be elaborated and produce new insights of the Feynman graph, for instance branch cuts or singularities representing particle productions.

\section{Examples}

In this section we explain our method by means of three different examples, the one-, twoand three-loop banana diagram. This demonstrates how our general method is applied on explicit Feynman integrals and moreover shows the power of our method. For the reader the difficulty of our examples increases with the loop order and new appearing issues are highlighted and discussed case by case.

\subsection{Example 1: the bubble graph}

As the first example we discuss the one-loop banana diagram which is also called the bubble graph. This Feynman diagram can also be calculated directly with usual Feynman graph techniques [44]. Nevertheless, we will use for pedagogical reasons the bubble graph to introduce our method.

In our conventions the bubble integral is defined as

$$
\mathcal{F}_{\sigma_{1}}\left(t, \xi_{1}, \xi_{2}\right)=\int_{x, y \geq 0} \frac{x \mathrm{~d} y-y \mathrm{~d} x}{x y\left(t-\left(\xi_{1}^{2} x+\xi_{2} y^{2}\right)\left(\frac{1}{x}+\frac{1}{y}\right)\right)}=-\xi_{1} \xi_{2} \int_{x, y \geq 0} \frac{x \mathrm{~d} y-y \mathrm{~d} x}{x^{2}+u x y+y^{2}}
$$

where in the second line the coordinates are rescaled and $u=\frac{\xi_{1}^{2}+\xi_{2}^{2}-t}{\xi_{1} \xi_{2}}$ is introduced. 
Figure 2. The toric diagram for the bubble graph.

Following our method we associate to the bubble graph (3.1) the polynomial constrain

$$
P_{1}=x^{2}+u x y+y^{2}
$$

in projective space $\mathbb{P}$. For generic values of the parameter $u$ this defines two different points in $\mathbb{P}$. It looks a bit artificial but we can give a toric description of this algebraic variety consisting of two points. We take the Newton polytope of (3.2) which is shown in figure 2.

It has a single $l$-vector and Batyrev coordinate

$$
l=(-2 ; 1,1) \quad \text { and } \quad z=\frac{1}{u^{2}} .
$$

As explained in section 2 we expect two functions spanning the function space of the bubble graph. One is coming from the maximal cut integral and the other one is a special solution of the inhomogeneous differential equation corresponding to the bubble graph. Furthermore, there is only a single true parameter for which we take naturally the Batyrev coordinate $z$ from (3.3).

The holomorphic period can be computed directly from the integral or from the $l$ vector $(3.3)$

$$
\begin{aligned}
\varpi & =\frac{1}{2 \pi i} \int_{S^{1}} \frac{x \mathrm{~d} y-y \mathrm{~d} x}{\sqrt{z}\left(x^{2}+y^{2}\right)+x y}=-\frac{1}{2 \pi i} \int_{S^{1}} \frac{1}{1+\sqrt{z}\left(v+\frac{1}{v}\right)} \frac{\mathrm{d} v}{v} \\
& =-\frac{1}{2 \pi i} \int_{S^{1}} \sum_{n=0}^{\infty} \sum_{m=0}^{n}(-1)^{n}\left(\begin{array}{c}
n \\
m
\end{array}\right) z^{n / 2} v^{2 m-n} \frac{\mathrm{d} v}{v}=-\sum_{n=0}^{\infty} \frac{(2 n) !}{(n !)^{2}} z^{n}=-\frac{1}{\sqrt{1-4 z}},
\end{aligned}
$$

where we have introduced the variable $v=\frac{x}{y}$. Moreover, $\varpi$ satisfies the first order differential equation

$$
\mathcal{D} \varpi=(1-4 z) \theta \varpi-2 z \varpi=0
$$

with the logarithmic derivative $\theta=z \partial_{z}$.

Now we apply the operator $\mathcal{D}$ from (3.5) on the integrand of the geometrical chain integral (2.34) containing the inner point of the polytope $u$ expressed through the Batyrev coordinate $z$. At the end we relate this expression to the bubble graph simply by dividing through the inner point. Fortunately, the integral in the bubble case can be computed analytically

$$
\mathcal{D} \Pi_{\sigma_{1}}=\mathcal{D} \int_{x, y \geq 0} u \frac{x \mathrm{~d} y-y \mathrm{~d} x}{x^{2}+u x y+y^{2}}=\int_{x, y \geq 0} \mathcal{D} \frac{x \mathrm{~d} y-y \mathrm{~d} x}{\sqrt{z}\left(x^{2}+y^{2}\right)+x y}=1 .
$$

This extends the homogeneous differential equation (3.5) to an inhomogeneous one

$$
(1-4 z) \theta \Pi_{\sigma_{1}}(z)-2 z \Pi_{\sigma_{1}}(z)=1
$$


A special solution to this inhomogeneous differential equation is given by

$$
\varpi_{S}=\varpi \log (z)+2 z+7 z^{2}+\frac{74}{3} z^{3}+\frac{533}{3} z^{4}+\cdots .
$$

Then the general solution to the inhomogeneous differential equation (3.7) is given by $\Pi_{\sigma_{1}}=\varpi_{S}+\lambda \varpi$ with $\lambda \in \mathbb{C}$. We can relate this solution to the bubble graph by dividing with the inner point $u$ and rescaling it by $-\xi_{1} \xi_{2}$. The parameter $\lambda$ can be fixed by calculating the bubble graph (3.1) at a special point in moduli space, for example $u=1$.

In the literature [45] the $l$-loop banana diagrams were analyzed in the equal mass case, i.e. $\xi_{i}=1$ for $i=1, \ldots l+1$. The one-loop bubble diagram satisfies the inhomogeneous first order equation

$$
t(t-4) f_{1}^{\prime}(t)+(t-2) f_{1}(t)=-2 !
$$

After dividing $\Pi_{\sigma_{1}}$ by the inner point this is exactly the differential equation it satisfies.

\subsection{Example 2: the sunset graph}

Our second example deals with the two-loop Banana diagram also known as the sunset graph. A different discussion of the sunset graph is given in [18] from which we adopt parts of our notation.

The sunset Feynman graph is defined by

$$
\mathcal{F}_{\sigma_{2}}\left(t, \xi_{1}, \xi_{2}, \xi_{3}\right)=\int_{\sigma_{2}} \frac{\mu_{2}}{P_{2}\left(t, \xi_{1}, \xi_{2}, \xi_{3} ; x\right)}=\int_{\sigma_{2}} \frac{x \mathrm{~d} y \wedge \mathrm{d} z-y \mathrm{~d} x \wedge \mathrm{d} z+z \mathrm{~d} x \wedge \mathrm{d} y}{x y z\left(t-\left(\xi_{1}^{2} x+\xi_{2}^{2} y+\xi_{3}^{2} z\right)\left(\frac{1}{x}+\frac{1}{y}+\frac{1}{z}\right)\right)},
$$

with the integration domain defined in (2.2). It can be interpreted as a relative period on an elliptic curve defined by the polynomial constraint

$$
P_{2}=t x y z-\xi_{1}^{2} x^{2} y-\xi_{1}^{2} x^{2} z-\xi_{1}^{2} x y z-\xi_{2}^{2} x y^{2}-\xi_{2}^{2} x y z-\xi_{2}^{2} y^{2} z-\xi_{3}^{2} x y z-\xi_{3}^{2} x z^{2}-\xi_{3}^{2} y z^{2}
$$

in an ambient space given by two-dimensional projective space $\mathbb{P}^{2}$ as explained in section 2.2. Our approach is strongly based on this geometric interpretation. For convenience we rescale the coordinates and introduce a simpler parametrization of the elliptic curve. The polynomial is then given as

$$
P_{2}=x y^{2}+y z^{2}+x^{2} z+m_{1} x z^{2}+m_{2} x^{2} y+m_{3} y^{2} z+u x y z .
$$

We notice that the polynomial (3.12) describes the blow up of $\mathbb{P}^{2}$ in three points which we call in the following $\mathcal{E}_{B 3}$. In [20] a nice analysis of the different blow ups of $\mathbb{P}^{2}$ is carried out from which we can extract same information for the toric description. In figure 3 the polyhedron corresponding to (3.12) is shown. The polyhedron's vertices are given by

$$
\nu_{2}=\{(0,-1),(1,0),(1,1),(0,1),(-1,0),(-1,-1),(0,0)\} .
$$




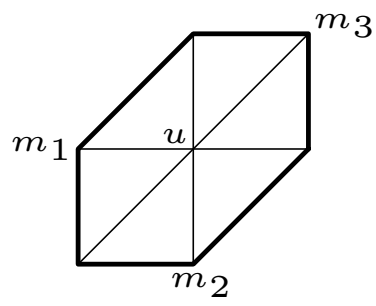

Figure 3. Toric diagram for the sunset graph.

The corresponding Mori cone generators are given by

$$
\begin{array}{ll}
\tilde{l}_{1}=(1,-1,1,0,0,0,-1), & \tilde{l}_{2}=(0,1,-1,1,0,0,-1) \\
\tilde{l}_{3}=(0,0,1,-1,1,0,-1), & \tilde{l}_{4}=(0,0,0,1,-1,1,-1) \\
\tilde{l}_{5}=(1,0,0,0,1,-1,-1), & \tilde{l}_{6}=(-1,1,0,0,0,1,-1)
\end{array}
$$

generating a non-simplicial cone. From the general discussion in section 2.2.4 we only need four independent $l$-vectors and also that in all columns of (3.14) except the one corresponding to the inner point there is at least one positive entry. This does still not yield a distinct choice of four $l$-vectors but all of them can be used. We take for our collection of four $l$-vectors the ones which restrict to the Mori cone generators of the cubic in $\mathbb{P}^{2}$ and the other blow ups of $\mathbb{P}^{2}$ in one and two points. So we take in the following the four $l$-vectors

$$
l_{1}=\tilde{l}_{1}, \quad l_{2}=\tilde{l}_{2}, \quad l_{3}=\tilde{l}_{3} \quad \text { and } \quad l_{4}=\tilde{l}_{4}
$$

Toric geometry singles out a natural choice of parametrization of the algebraic variety given by the Batyrev coordinates (2.16). These parameters are related to the ones in (3.11) and (3.12) by

$$
\begin{aligned}
z_{1}=-\frac{m_{2} m_{3}}{u}=-\frac{\xi_{1}^{2}}{\xi_{1}^{2}+\xi_{2}^{2}+\xi_{3}^{2}-t}, & z_{2}=-\frac{1}{u m_{3}}=-\frac{\xi_{3}^{2}}{\xi_{1}^{2}+\xi_{2}^{2}+\xi_{3}^{2}-t} \\
z_{3}=-\frac{m_{1} m_{3}}{u}=-\frac{\xi_{2}^{2}}{\xi_{1}^{2}+\xi_{2}^{2}+\xi_{3}^{2}-t}, & z_{4}=-\frac{1}{u m_{1}}=-\frac{\xi_{1}^{2}}{\xi_{1}^{2}+\xi_{2}^{2}+\xi_{3}^{2}-t} .
\end{aligned}
$$

Upon collecting the main toric information of our problem we can start with our strategy. The first part of our strategy will be the computation of the periods corresponding to the maximal cut integral.

Let us note here in passing that in the elliptic curve case it is not necessary to solve any differential equation to obtain the period integrals and hence the mass dependence of the maximal cut integral. The periods are completely determined by modular functions as follows from [20]: we can bring the constraint $P_{2}=0$ (3.12) defining the elliptic curve into Weierstrass form $y^{2}=4 x^{3}-x g_{2}(u, \underline{m})-g_{3}(u, \underline{m})$. This defines the modular parameter $\tau(u, \underline{m})$ from the definition of the Hauptmodul $j$ of $\operatorname{PSL}(2, \mathbb{Z})$ as

$$
\frac{1728 g_{2}^{3}(u, \underline{m})}{g_{2}^{3}(u, \underline{m})-27 g_{3}^{2}(u, \underline{m})}=j=\frac{1}{q}+744+192688 q+21493760 q^{2}+\mathcal{O}\left(q^{3}\right),
$$


where $q=\exp (2 \pi i \tau)$. Then the period $\int_{a} \Omega / u$ which yields the maximal cut integral is given in terms of the Eisenstein series as

$$
\partial_{u} t(u)=\int_{a} \Omega=\sqrt{\frac{E_{4}(\tau(u, \underline{m})) g_{3}(u, \underline{m})}{E_{6}(\tau(u, \underline{m})) g_{2}(u, \underline{m})}} .
$$

Moreover, the dual period $\int_{b} \Omega$ can be obtained by special geometry of non-compact threefolds as $\left.\partial_{t} \int_{b} \Omega=-\frac{1}{2 \pi i} \tau(u, \underline{m})\right)=\partial_{t}^{2} F(t)$, where $F$ is the prepotential that features in local mirror symmetry as generating function for the genus zero BPS invariants $n_{0}^{\beta}$, which is given by

$$
F(Q)=-\frac{c^{i j k}}{3 !} t_{i} t_{j} t_{k}+\frac{c^{i j}}{2} t_{i} t_{j}+c^{i} t_{i}+c \sum_{\beta \in H_{2}(W, \mathbb{Z})} n_{0}^{\beta} \operatorname{Li}_{3}\left(Q^{\beta}\right) .
$$

Here $t_{i}$ are the flat coordinates, $Q_{i}=\exp \left(t_{i} / 2 \pi i\right)$ and the $c^{*}$ are classical intersection numbers on the mirror $W$. In [20] the Kähler classes $t_{i}$ for $i=1, \ldots, 4$ of the mirror have been identified. These are linearly related to the Batyrev coordinates (3.16). With $Q_{i}=\exp \left(t_{i} / 2 \pi i\right)$ they relate to the physical parameters as

$$
Q=\left(Q_{1} Q_{2} Q_{3} Q_{4}\right)^{\frac{1}{3}}, \quad m_{1}=\frac{\left(Q_{1} Q_{3} Q_{4}\right)^{\frac{1}{3}}}{Q_{2}^{\frac{2}{3}}}, \quad m_{2}=\frac{\left(Q_{1} Q_{2} Q_{4}\right)^{\frac{1}{3}}}{Q_{3}^{\frac{2}{3}}}, \quad m_{3}=\frac{\left(Q_{1} Q_{2} Q_{3}\right)^{\frac{1}{3}}}{Q_{4}^{\frac{2}{3}}} .
$$

This allows to relate the full integer genus zero BPS expansion $n_{0}^{\beta}$ in the four Kähler parameters [20]

$$
\begin{aligned}
F= & \text { cl. }+L_{0,0,0,1}+L_{1,0,0,1}-2 L_{1,0,1,1}+3 L_{1,1,1,1}+3 L_{2,1,1,1}-4 L_{2,1,1,2}+5 L_{2,1,2,2} \\
& -6 L_{2,2,2,2}+5 L_{3,1,2,2}-6 L_{3,1,2,3}+7 L_{3,1,3,3}-36 L_{3,2,2,2}+35 L_{3,2,2,3}-32 L_{3,2,3,3} \\
& +27 L_{3,3,3,3}+7 L_{4,1,3,3}-8 L_{4,1,3,4}+9 L_{4,1,4,4}-6 L_{4,2,2,2}+35 L_{4,2,2,3} \\
& -32 L_{4,2,2,4}-160 L_{4,2,3,3}+135 L_{4,2,3,4}-110 L_{4,2,4,4}+531 L_{4,3,3,3} \\
& -400 L_{4,3,3,4}+286 L_{4,3,4,4}-192 L_{4,4,4,4}+\operatorname{Sym}_{i j k}\left(L_{a, i, j, k}\right)+\cdots
\end{aligned}
$$

to the full set of physical parameters. Here $L_{\beta}:=\operatorname{Li}_{3}\left(\prod_{i=1}^{4} Q_{i}^{\beta_{i}}\right)$. In [39] BPS invariants are given for the projective parametrization $n_{i j k}$. The relation to the geometrical BPS invariants is $\sum_{a} n_{0}^{a i j k}=n_{i j k}$. It is clear from (3.20) and the symmetries of the polytop that the last formula is symmetric in the $i j k$ indices. Moreover, the one parameter specialization also noted in [39] is given by $n_{d}=\sum_{a, i+j+k=d} n_{0}^{a i j k}$. While we think that in the elliptic two-loop case this relation of the BPS expansion to the Feynman graph is remarkable but not very useful, it becomes more useful for the higher loop banana graphs as we explain in section 3.3.3.

\subsubsection{The sunset maximal cut integral}

The maximal cut integral of the sunset graph $\mathcal{F}_{T^{2}}\left(t, \xi_{1}, \xi_{2}, \xi_{3}\right)$ is defined by replacing the simplex $\sigma_{2}$ by a torus $T^{2}$. Instead of focusing on the maximal cut Feynman graph we rather deal with the related geometrical period which includes additionally the inner point $u$ of the toric diagram. The expression

$$
\Pi\left(T^{2}\right)\left(u, m_{1}, m_{2}, m_{3}\right)=\int_{T^{2}} u \frac{x \mathrm{~d} y \wedge \mathrm{d} z-y \mathrm{~d} x \wedge \mathrm{d} z+z \mathrm{~d} x \wedge \mathrm{d} y}{x y^{2}+y z^{2}+x^{2} z+m_{1} x z^{2}+m_{2} x^{2} y+m_{3} y^{2} z+u x y z}
$$


describes a "usual" period on the elliptic curve $\mathcal{E}_{B 3}$ and it is easily related to the maximal cut integral $\mathcal{F}_{T^{2}}$ by dividing with $u$. At the point of maximal unipotent monodromy the geometrical period $\Pi\left(T^{2}\right)=\varpi$ is given by a single holomorphic power series (2.21). Evaluating the period (3.22) at a generic point in moduli space requires the knowledge of a period basis. Such a period basis can be found as follows: homology theory of a generic elliptic curve tells us that there exists only a pair of one-cycles, i.e. $H_{2}\left(T^{2}\right)=\mathbb{Z}^{2}$. So if we take the $(1,0)$-form $\frac{a_{0} \mu_{2}}{P_{2}}$ with $a_{0}$ the inner point of the polytope and $P_{2}$ the hypersurface constraint defining the elliptic curve there are only two independent periods. Here it is important to remark that for elliptic curves this statement is independent of the parametrization, in particular, independent of the number of moduli. For the geometrical period $\Pi\left(T^{2}\right)$ and therefore also for the maximal cut integral $\mathcal{F}_{T^{2}}$ this means that there are two independent functions which linearly combined yield (3.22) at a generic point in moduli space.

In our toric analysis it is convenient to use the Batyrev parameters defined in (3.16). Later we will see that the usage of this particular choice of parametrization enables us to fully determine the sunset graph. Moreover, it simplifies many of the subsequent results.

From the Mori cone generators (3.15) one can directly write down the holomorphic period at the point of maximal unipotent monodromy given by

$$
\begin{aligned}
\varpi(\underline{z})= & \sum_{\underline{m} \geq 0} \frac{\Gamma\left(1+m_{1}+m_{2}+m_{3}+m_{4}\right)}{\Gamma\left(1+m_{1}\right) \Gamma\left(1-m_{1}+m_{2}\right) \Gamma\left(1+m_{1}-m_{2}+m_{3}\right) \Gamma\left(1+m_{3}-m_{4}\right)} \\
& \cdot \frac{1}{\Gamma\left(1+m_{4}\right) \Gamma\left(1+m_{2}-m_{3}+m_{4}\right)} z_{1}^{m_{1}} z_{2}^{m_{2}} z_{3}^{m_{3}} z_{4}^{m_{4}}
\end{aligned}
$$

with the abbreviations $\underline{z}=\left(z_{1}, z_{2}, z_{3}, z_{4}\right)$ and $\underline{m}=\left(m_{1}, m_{2}, m_{3}, m_{4}\right)$. This is the most generic four-parameter holomorphic period of $\mathcal{E}_{B 3}$. The geometrical period (3.22) has one less parameter since one-parameter can be scaled away. Therefore, we have to specialize the four-parameter solution (3.23) to a three-parameter one. We remark that from (3.16) the parameters $z_{1}$ and $z_{4}$ have the same value if expressed in the physical parameters. This means that the four-parameter solution (3.23) specialized on the subslice with $z_{1}=z_{4}$ corresponds to the holomorphic solution of the geometrical period (3.22) at the maximal unipotent monodromy point.

This subslice is not as problematic as for the higher loop banana graphs because the sum over $m_{4}$ still contains a parameter, here $z_{1}$. But still we can use the $\Gamma$-functions in (3.23) to bound the summation over the index $m_{4}$ by $m_{3}-m_{4} \geq 0$. We obtain for the first few orders

$$
\begin{aligned}
\varpi\left(z_{1}, z_{2}, z_{3}\right)= & 1+2 z_{1} z_{2}+2 z_{1} z_{3}+2 z_{2} z_{3}+12 z_{1} z_{2} z_{3} \\
& +6 z_{1}^{2} z_{2}^{2}+24 z_{1}^{2} z_{2} z_{3}+24 z_{1} z_{2}^{2} z_{3}+6 z_{1}^{2} z_{3}^{2}+24 z_{1} z_{2} z_{3}^{2}+6 z_{2}^{2} z_{3}^{2}+\cdots
\end{aligned}
$$

Now our strategy is as follows: we compute the holomorphic solution to high order such that we can find a set of differential operators annihilating it. This set of differential operators has to be complete in a sense that its solutions form a basis of period integrals on the elliptic curve $\mathcal{E}_{B 3}$. Therefore, a suitable ansatz for these operators is crucial. Again 
homology theory of the elliptic curve tells us what kind of solutions we expect and so the rare form of the operators. For $\mathcal{E}_{B 3}$ only two solutions exist. At the point of maximal unipotent monodromy the analytic structure of them is also known. One is a holomorphic function in the parameters and the other contains single logarithms of the parameters. For the differential operator ideal this implies that we are searching for first order operators in the parameters $\left(z_{1}, z_{2}, z_{3}\right)$. Having found the first few operators one has to increase the number of operators until they are enough to fully determine the two different periods. As a possible generating set of the ideal we find

$$
\begin{aligned}
\mathcal{D}_{1}= & \theta_{1}-\theta_{2}+z_{2}\left(\theta_{1}-\theta_{2}+\theta_{3}+2 z_{3}\left(\theta_{1}+\theta_{2}+\theta_{3}+1\right)\right) \\
& -z_{1}\left(-\theta_{1}+\theta_{2}+\theta_{3}+2 z_{3}\left(\theta_{1}+\theta_{2}+\theta_{3}+1\right)\right) \\
\mathcal{D}_{2}= & \theta_{2}-\theta_{3}+z_{3}\left(\theta_{1}+\theta_{2}-\theta_{3}\right)-z_{2}\left(\theta_{1}-\theta_{2}+\theta_{3}\right)-2 z_{1}\left(z_{2}-z_{3}\right)\left(\theta_{1}+\theta_{2}+\theta_{3}+1\right) \\
\mathcal{D}_{3}= & \left(\theta_{1}-\theta_{2}\right)\left(\theta_{1}+\theta_{2}-\theta_{3}\right)+z_{1}\left(\theta_{1}-\theta_{2}-\theta_{3}\right)\left(\theta_{1}+\theta_{2}+\theta_{3}+1\right) \\
& +z_{2}\left(\theta_{1}-\theta_{2}+\theta_{3}\right)\left(\theta_{1}+\theta_{2}+\theta_{3}+1\right)
\end{aligned}
$$

with $\theta_{i}=z_{i} \partial_{z_{i}}$ for $i=1,2,3$. The missing period is then given by

$$
\Pi\left(\Gamma_{1}\right)\left(z_{1}, z_{2}, z_{3}\right)=\varpi\left(\log \left(z_{1}\right)+\log \left(z_{2}\right)+\log \left(z_{3}\right)\right)+\Sigma_{1}
$$

with

$$
\begin{aligned}
\Sigma_{1}= & z_{1}+z_{2}+z_{3}-\frac{z_{1}^{2}}{2}+7 z_{1} z_{2}+7 z_{1} z_{3}-\frac{z_{2}^{2}}{2}+7 z_{2} z_{3}-\frac{z_{3}^{2}}{2} \\
& +\frac{z_{1}^{3}}{3}+3 z_{1}^{2} z_{2}+3 z_{1}^{2} z_{3}+3 z_{1} z_{2}^{2}+3 z_{1} z_{3}^{2}+48 z_{1} z_{2} z_{3}+\frac{z_{2}^{3}}{3}+\frac{z_{3}^{3}}{3} \\
& +3 z_{2} z_{3}^{2}+3 z_{2}^{2} z_{3}+\cdots .
\end{aligned}
$$

These two solutions (3.24) and (3.26) form a basis of the periods for the elliptic curve $\mathcal{E}_{B 3}$. Using the relations (3.16) we can divide by the inner point and transform this basis to the necessary point in moduli space such that they can be linearly combined to yield the maximal cut integral $\mathcal{F}_{T^{2}}$.

In the next section we extend the differential operator ideal (3.25) such that it governs all functions describing the full geometrical sunset Feynman graph $\Pi_{\sigma_{2}}$. By dividing with the inner point we can transfer these results to the actual Feynman integral (3.10).

\subsubsection{Extension to inhomogeneous differential operators}

As explained in section 2.3 we find as the first step the inhomogeneities of the operators (3.25). Again we use the Batyrev coordinates $\left(z_{1}, z_{2}, z_{3}\right)$ which is crucial for the applicability of our method. We apply the operators (3.25) on the geometrical differential $\frac{u \mu_{2}}{P_{2}}$ and integrate afterwards over the two-dimensional simplex $\sigma_{2}$. These chain integrals can not in general be computed analytically with generic parameters but numerical evaluations of these integrals for fixed values of the parameters are possible. Now the advantage of the Batyrev coordinates is that we can guess the exact values of the numerical results. 
We claim that the differential operator ideal only produces simple logarithmic expressions in the Batyrev coordinates $\left(z_{1}, z_{2}, z_{3}\right)$. For $(3.25)$ we find the following inhomogeneities ${ }^{18}$

$$
\begin{aligned}
& \mathcal{D}_{1} \Pi_{\sigma_{2}}=-\log \left(z_{2}\right)+\log \left(z_{3}\right) \\
& \mathcal{D}_{2} \Pi_{\sigma_{2}}=-\log \left(z_{1}\right)+\log \left(z_{2}\right) \\
& \mathcal{D}_{3} \Pi_{\sigma_{2}}=0 .
\end{aligned}
$$

We think that in another parametrization, for instance the physical parameters $\left(t, \xi_{1}, \xi_{2}, \xi_{3}\right)$, and without the inner point these integrals can neither be computed analytically nor their numerical values can be guessed. Only the geometrical differential in the special parametrization with the Batyrev parameters guarantees the feasibility of our method.

Having found the complete set of inhomogeneous differential operators their solutions can be computed easily. One has to extend the solutions of the homogeneous system (3.25) by a special solution satisfying (3.28). As an ansatz for this solution we increase the power of logarithms in $\left(z_{1}, z_{2}, z_{3}\right)$ up to two. Then we find as a possible choice of special solution

$$
\begin{aligned}
\varpi_{S}\left(z_{1}, z_{2}, z_{3}\right)= & \left(\log \left(z_{1}\right) \log \left(z_{2}\right)+\log \left(z_{1}\right) \log \left(z_{3}\right)+\log \left(z_{2}\right) \log \left(z_{3}\right)\right) \varpi_{0}+2 \log \left(z_{1}\right) \\
& +2 \log \left(z_{2}\right)+2 \log \left(z_{3}\right)+2 z_{1} \log \left(z_{1}\right)+2 z_{2} \log \left(z_{2}\right)+2 z_{3} \log \left(z_{3}\right) \\
& -\frac{z_{1}^{2}}{2}+10 z_{1} z_{2}-\frac{z_{2}^{2}}{2}+10 z_{1} z_{3}-\frac{z_{3}^{2}}{2}-z_{1}^{2} \log \left(z_{1}\right)+10 z_{1} z_{2} \log \left(z_{1}\right) \\
& +10 z_{1} z_{3} \log \left(z_{1}\right)+6 z_{2} z_{3} \log \left(z_{1}\right)+10 z_{1} z_{2} \log \left(z_{2}\right)-z_{2}^{2} \log \left(z_{2}\right) \\
& +6 z_{1} z_{3} \log \left(z_{2}\right)+10 z_{2} z_{3} \log \left(z_{2}\right)+6 z_{1} z_{3} \log \left(z_{3}\right) \\
& +10 z_{1} z_{3} \log \left(z_{3}\right)+10 z_{2} z_{3} \log \left(z_{3}\right)-z_{3}^{2} \log \left(z_{3}\right)+\cdots
\end{aligned}
$$

The general solution is then a linear combination of the form $\Pi_{\sigma_{2}}=\varpi_{S}+\lambda_{0} \varpi+\lambda_{1} \Pi\left(\Gamma_{1}\right)$ with $\lambda_{0}, \lambda_{1} \in \mathbb{C}$. We can express $\Pi_{\sigma_{2}}$ through the physical parameters $\left(t, \xi_{1}, \xi_{2}, \xi_{3}\right)$ and divide it by the inner point to find the full sunset Feynman graph $\mathcal{F}_{\sigma_{2}}(3.10)$.

\subsubsection{Comparison with the equal mass case and other known results}

Many results about the sunset graph are already known in the literature [35, 46]. In particular, the equal mass case meaning $\xi_{i}=1$ for $i=1,2,3$ was analyzed many times. In this case, the maximal cut integral is up to a factor of $u=t-3$ (2.17) the holomorphic period of the Barth-Nieto elliptic curve that can be represented as in (2.18). The equal mass sunset graph has to satisfy an inhomogeneous second order differential equation [45] in the momentum variable $t$

$$
t(t-1)(t-9) f_{2}^{\prime \prime}(t)+\left(3 t^{2}-20 t+9\right) f_{2}^{\prime}(t)+(t-3) f_{2}(t)=-3 ! .
$$

\footnotetext{
${ }^{18}$ We checked this numerically up to more than 15 digits and for different values of the variables $z_{i}$ for $i=1,2,3$.
} 


\begin{tabular}{|c|ccc|}
\hline$\Pi_{\sigma_{2}}=\lambda_{S} \varpi_{S}+\lambda_{0} \varpi_{0}+\lambda_{1} \Pi\left(\Gamma_{1}\right)$ & $\lambda_{S}$ & $\lambda_{0}$ & $\lambda_{1}$ \\
\hline order 5 & 0.9998 & $-29.6275+42.7536 i$ & $-13.6122-18.8466 i$ \\
order 10 & 1.0000 & $-29.6088+42.7407 i$ & $-13.6048-18.8496 i$ \\
\hline order 5 & $1.0004+0.0007 i$ & $70.0913+109.3340 i$ & $-34.7859-18.8389 i$ \\
order 10 & $1.0004+0.0007 i$ & $70.0913+109.3340 i$ & $-34.7859-18.8389 i$ \\
\hline
\end{tabular}

Table 1. Linear combination of solutions for the sunset graph. In the first two rows are the values for our solutions whereas the last two give the ones for the solutions from [18].

Our three-parameter solutions (3.24), (3.26) and (3.29) break down in the equal mass case $^{19}$ to the solutions of (3.30). This shows that they reproduces the well established equal mass results.

For the sunset graph a second test is possible since in [18] an inhomogeneous differential equation in all physical parameters is given which the sunset graph has to satisfy. Here we notice that our holomorphic and single logarithmic solutions expressed in the physical parameters fulfill this equation. The special solution (3.29) does not. A direct comparison between our special solution and the solutions to the inhomogeneous differential equation in [18] shows that the discrepancy between them is only in the terms having no logarithm in the variable $s=1 / t$. Such a small difference can be a result of a typo in the polynomials given in [18] but a general mistake in their derivation of the inhomogeneous differential equation can not be excluded.

To demonstrate the correctness of our solutions we made some numerical checks. We evaluated the sunset Feynman graph (3.10) at three different points ${ }^{20}$ to fix the linear combination of our three solutions. ${ }^{21}$ Having found the right combination of solutions given in table 1 we checked for further values of the parameters and compare the precision for different expansion orders of $\varpi, \Pi\left(\Gamma_{1}\right)$ and $\varpi_{S}$. Our results are listed in table 2. Notice, that it is important that the value of one $\xi_{i}$ is fixed since there are only three physical degrees of freedom after rescaling. We choose $\xi_{3}$ to be fixed. With increasing expansion order our solutions fit better and better to the sunset graph which we could not observe for the solutions of [18]. Moreover, the factor $\lambda_{S}$ of the special solution $\varpi_{S}$ tends to the value one as expected.

\subsection{Example 3: the three-loop banana graph}

As our last and most complicated example we demonstrate the applicability of our approach for the three-loop banana diagram

$$
\begin{aligned}
\mathcal{F}_{\sigma_{3}}\left(t, \xi_{1}, \xi_{2}, \xi_{3}, \xi_{4}\right) & \\
& =\int_{\sigma_{3}} \frac{x \mathrm{~d} y \wedge \mathrm{d} z \wedge \mathrm{d} w-y \mathrm{~d} x \wedge \mathrm{d} z \wedge \mathrm{d} w+z \mathrm{~d} x \wedge \mathrm{d} y \wedge \mathrm{d} w-w \mathrm{~d} x \wedge \mathrm{d} y \wedge \mathrm{d} z}{x y z w\left(t-\left(\xi_{1}^{2} x+\xi_{2}^{2} y+\xi_{3}^{2} z+\xi_{4}^{2} w\right)\left(\frac{1}{x}+\frac{1}{y}+\frac{1}{z}+\frac{1}{w}\right)\right)} .
\end{aligned}
$$

\footnotetext{
${ }^{19}$ Notice that before one can apply the differential equation (3.30) on our solutions they have to be transformed at the same point in moduli space, which is here $t \mapsto \frac{1}{t}$.

${ }^{20} \mathrm{We}$ took for the three points the values $\left(s, \xi_{1}, \xi_{2}, \xi_{3}\right)=\left(s_{1}+i / 10,1 / 10,1 / 20,1 / 30\right)$, for $s_{1}=1 / 10, s_{2}=$ $1 / 20$ and $s_{3}=1 / 30$.

${ }^{21}$ We fixed our basis of solutions such that the holomorphic solution starts with one and the constant piece in the single logarithmic solution is zero. Moreover, we fixed the special solution by requiring that the constant term and the constant term multiplied by $\log s$ is vanishing.
} 


\begin{tabular}{|c|cc|cc|}
\hline$s, \xi_{1}, \xi_{2}, \xi_{3}$, & order 5 & order 10 & order 5 & order 10 \\
\hline $1 / 27+\mathrm{i} / 20,1 / 10,1 / 20,1 / 30$ & $9 \cdot 10^{-5}$ & $5 \cdot 10^{-9}$ & $2 \cdot 10^{-4}$ & $2 \cdot 10^{-4}$ \\
$1 / 21+\mathrm{i} / 10,1 / 10,1 / 50,1 / 30$ & $4 \cdot 10^{-4}$ & $6 \cdot 10^{-9}$ & 30 & 30 \\
$1 / 24+\mathrm{i} / 10,1 / 10+\mathrm{i} / 15,1 / 20,1 / 30$ & $6 \cdot 10^{-4}$ & $5 \cdot 10^{-9}$ & 22 & 22 \\
\hline
\end{tabular}

Table 2. The table shows how precise the relative periods combined as listed in table 1 describe the Feynman graph. We show the absolute value of the difference between the numerical computation of the sunset graph and the evaluation of the linear combination of solutions. Increasing the expansion order increases the precision of our results given as the second and third column. The last columns give the results from [18] which do not increase their precision.

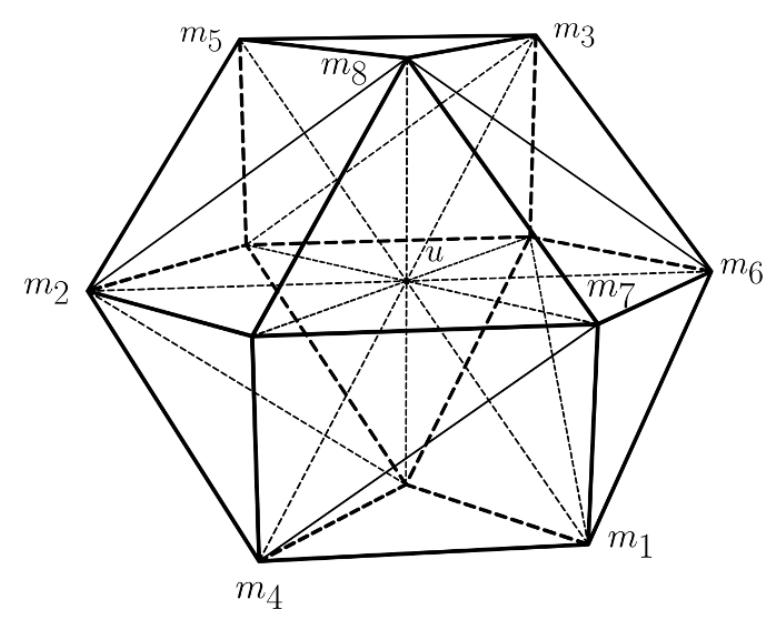

Figure 4. Toric diagram for the three-loop banana graph

The three-loop banana Feynman graph (3.31) can again be interpreted as a relative period now on a K3 surface. This K3 surface is defined by the constraint $P_{3}$ from the denominator in (3.31). After a rescaling of the coordinates we obtain

$$
\begin{aligned}
P_{3}= & x^{2} y z+x y w^{2}+x z w^{2}+y z w^{2}+m_{1} x y^{2} w+m_{2} x^{2} z w+m_{3} y z^{2} w+m_{4} x^{2} y w \\
& +m_{5} x z^{2} w+m_{6} y^{2} z w+m_{7} x y^{2} z+m_{8} x y z^{2}+u x y z w .
\end{aligned}
$$

The polytope $P_{\Delta_{3}}$ corresponding to the banana graph together with a triangulation is shown in figure 4 . Its vertices are given by

$$
\begin{gathered}
\nu_{3}=\{(-1,1,0),(1,0,0),(0,-1,1),(0,0,1),(1,-1,0),(1,0,-1),(0,0,-1),(-1,0,1), \\
(0,-1,0),(-1,0,0),(0,1,0),(0,1,-1),(0,0,0)\} .
\end{gathered}
$$

Furthermore, the Mori cone generators corresponding to the triangulation drawn in the polytope in figure 4 are given by

$$
\begin{aligned}
& l_{1}=(0,0,-1,0,1,0,0,1,0,0,0,0,-1), \quad l_{2}=(0,-1,0,0,1,0,0,0,0,0,1,0,-1) \\
& l_{3}=(0,-1,0,1,0,1,0,0,0,0,0,0,-1), \quad l_{4}=(0,0,-1,1,0,0,0,0,1,0,0,0,-1) \\
& l_{5}=(-1,0,0,-1,0,0,0,1,0,0,1,0,0), \quad l_{6}=(0,0,0,0,-1,1,-1,0,1,0,0,0,0)
\end{aligned}
$$




$$
\begin{aligned}
& l_{7}=(0,0,1,0,0,0,0,-1,-1,1,0,0,0), \quad l_{8}=(0,1,0,0,0,-1,0,0,0,0,-1,1,0) \\
& l_{9}=(1,0,0,0,0,0,1,0,0,-1,0,-1,0) .
\end{aligned}
$$

They form a simplicial Mori cone generated by $3^{2}$ vectors. For the subsequent discussion we need the Batyrev coordinates together with their relations to the physical paramters

$$
\begin{array}{rlrl}
z_{1}=-\frac{m_{2} m_{3}}{m_{5} u}=-\frac{\xi_{1}^{2}}{\xi_{1}^{2}+\xi_{2}^{2}+\xi_{3}^{2}+\xi_{4}^{2}-t}, & z_{5}=\frac{m_{3} m_{7}}{m_{6} m_{8}}=1 \\
z_{2}=-\frac{m_{2} m_{7}}{u}=-\frac{\xi_{2}^{2}}{\xi_{1}^{2}+\xi_{2}^{2}+\xi_{3}^{2}+\xi_{4}^{2}-t}, & z_{6}=\frac{m_{4}}{m_{2}}=1 \\
z_{3}=-\frac{m_{4} m_{8}}{u}=-\frac{\xi_{3}^{2}}{\xi_{1}^{2}+\xi_{2}^{2}+\xi_{3}^{2}+\xi_{4}^{2}-t}, & z_{7}=\frac{m_{5}}{m_{3}}=1 \\
z_{4}=-\frac{m_{8}^{2}}{m_{5} u}=-\frac{\xi_{4}^{2}}{\xi_{1}^{2}+\xi_{2}^{2}+\xi_{3}^{2}+\xi_{4}^{2}-t}, & z_{8}=\frac{m_{1}}{m_{4} m_{7}}=1 \\
z_{9}=\frac{m_{6}}{m_{1}}=1 .
\end{array}
$$

Having defined the most important information about the three-loop banana graph we want to find a set of functions describing it. We follow our general strategy but there are some subtleties which have not popped up for the sunset graph.

\subsubsection{Maximal cut integral}

As before, the maximal cut integral $\mathcal{F}_{T^{3}}\left(t, \xi_{1}, \xi_{2}, \xi_{3}, \xi_{4}\right)$ is related through the inner point to the K3 period integral

$$
\Pi\left(T^{3}\right)\left(u, m_{1}, m_{2}, m_{3}, m_{4}\right)=\int_{T^{3}} \frac{u \mu_{3}}{P_{3}} .
$$

We want to compute a basis for the periods on the K3 surface. Cohomology theory of the K3 surface can tell us again how many independent periods we expect. Differently as for elliptic curves the number of independent two-cycles depends on the number of moduli. For a $r$ parameter model we expect $r+2$ independent two-cycles and similarly $r+2$ independent periods. Moreover, the analytic structure of these periods can be specified further. There is exactly one holomorphic and one double logarithmic period on the K3. The remaining $r$ periods are single logarithmic ones.

The starting point of our method is the holomorphic period expressed through the Batyrev parameters which are much more as the physical parameters. From (3.35) five Batyrev parameters are set to one after identification with the physical parameters. The remaining four coordinates $\left(z_{1}, z_{2}, z_{3}, z_{4}\right)$ are related to the physical parameters and are such the only ones important in the following. From the Mori cone generators it is always possible to write down the general form of the holomorphic period but in all nine Batyrev parameters. We can expand this holomorphic solution in the "unphysical" parameters $\left(z_{5}, z_{6}, z_{7}, z_{8}, z_{9}\right)$ exactly and set them afterwards to one. This yields the holomorphic solution in the physically relevant four parameters. To insure that our expansion is exact in the unphysical parameters we use the particular form of the holomorphic periods in 
terms of $\Gamma$-functions. Since the numerator does never diverge for positive values of the index parameters $m_{i}, i=1, \ldots 9$ the $\Gamma$-functions in the denominator give bounds on the index parameters $m_{i}$. Concretely we obtain

$$
\begin{aligned}
\varpi\left(z_{1}, z_{2}, z_{3}, z_{4}\right)= & \sum_{\mathcal{M}} \frac{\Gamma\left(1+m_{1}+m_{2}+m_{3}+m_{4}\right)}{\Gamma\left(1+m_{3}+m_{4}-m_{5}\right) \Gamma\left(1+m_{1}+m_{2}-m_{6}\right) \Gamma\left(1+m_{1}+m_{5}-m_{7}\right) \Gamma\left(1+m_{4}+m_{6}-m_{7}\right)} \\
& \cdot \frac{z_{2}^{m_{2}} z_{3}^{m_{3}} z_{4}^{m_{4}} z_{5}^{m_{5}}}{\Gamma\left(1-m_{1}-m_{4}+m_{7}\right) \Gamma\left(1+m_{2}+m_{5}-m_{8}\right) \Gamma\left(1+m_{3}+m_{6}-m_{8}\right) \Gamma\left(1-m_{2}-m_{3}+m_{8}\right)} \\
& \cdot \frac{1}{\Gamma\left(1+m_{7}-m_{9}\right) \Gamma\left(1+m_{8}-m_{9}\right) \Gamma\left(1-m_{5}+m_{9}\right) \Gamma\left(1-m_{6}+m_{9}\right)}
\end{aligned}
$$

with the summation range given by

$$
\begin{aligned}
\mathcal{M}=\{0 \leq & m_{1} \leq \infty, 0 \leq m_{2} \leq \infty, 0 \leq m_{3} \leq \infty, 0 \leq m_{4} \leq \infty, 0 \leq m_{5} \leq m_{3}+m_{4} \\
& m_{2}+m_{3} \leq m_{8} \leq m_{2}+m_{5}, 0 \leq m_{6} \leq m_{1}+m_{2}, m_{1}+m_{4} \leq m_{7} \leq m_{1}+m_{5} \\
& \left.m_{6} \leq m_{9} \leq m_{7}\right\}
\end{aligned}
$$

We find

$$
\begin{aligned}
\varpi\left(z_{1}, z_{2}, z_{3}, z_{4}\right)= & 1+2\left(z_{1} z_{2}+z_{1} z_{3}+z_{1} z_{4}+z_{2} z_{3}+z_{2} z_{4}+z_{3} z_{4}\right) \\
& +12\left(z_{1} z_{2} z_{3}+z_{1} z_{2} z_{4}+z_{1} z_{3} z_{4}+z_{2} z_{3} z_{4}\right)+\cdots
\end{aligned}
$$

Then our strategy is the same as before. We expand the holomorphic solution (3.39) high enough that we can find a set of operators annihilating it. This time we are looking for second order operators in such a way that their solutions are given by a single holomorphic and a single double logarithmic solution and further four single logarithmic solutions. As a choice we take the operators $\mathcal{D}_{1}, \ldots, \mathcal{D}_{4}$ as generators for the differential operator ideal. They are listed in appendix A. Then a period basis is given by four single logarithmic solutions

$$
\begin{aligned}
& \Pi\left(\Gamma_{1}^{1}\right)=\varpi \log \left(z_{1}\right)+\Sigma_{1}^{1} \\
& \Pi\left(\Gamma_{1}^{2}\right)=\varpi \log \left(z_{2}\right)+\Sigma_{1}^{2} \\
& \Pi\left(\Gamma_{1}^{3}\right)=\varpi \log \left(z_{3}\right)+\Sigma_{1}^{3} \\
& \Pi\left(\Gamma_{1}^{4}\right)=\varpi \log \left(z_{4}\right)+\Sigma_{1}^{4},
\end{aligned}
$$

with

$$
\begin{aligned}
\Sigma_{1}^{1}= & -z_{1}+z_{2}+z_{3}+z_{4}+\frac{z_{1}^{2}}{2}+z_{1} z_{2}+z_{1} z_{3}+z_{1} z_{4}-\frac{z_{2}^{2}}{2}+z_{2} z_{3}+5 z_{2} z_{4}-\frac{z_{3}^{2}}{2}+5 z_{3} z_{4} \\
& -\frac{z_{4}^{2}}{2}-\frac{z_{1}^{3}}{3}-3 z_{1}^{2} z_{2}-3 z_{1}^{2} z_{3}-3 z_{1}^{2} z_{4}+3 z_{1} z_{2}^{2}+3 z_{1} z_{3}^{2}+3 z_{1} z_{4}^{2} \\
& +16 z_{1} z_{2} z_{3}+16 z_{1} z_{2} z_{4}+16 z_{1} z_{3} z_{4}+\frac{z_{2}^{3}}{3}+3 z_{2}^{2} z_{3}+3 z_{2}^{2} z_{4}+3 z_{2} z_{3}^{2}+3 z_{2} z_{4}^{2} \\
& +52 z_{2} z_{3} z_{4}+\frac{z_{3}^{3}}{3}+3 z_{3}^{2} z_{4}+3 z_{3} z_{4}^{2}+\frac{z_{4}^{3}}{3}+\cdots
\end{aligned}
$$


The other $\Sigma_{1}^{i}$ for $i=2,3,4$ are given as permutations, namely $\Sigma_{1}^{2}=\Sigma_{1}^{1}\left(z_{1} \leftrightarrow z_{2}\right), \Sigma_{1}^{3}=$ $\Sigma_{1}^{1}\left(z_{1} \leftrightarrow z_{3}\right)$ and $\Sigma_{1}^{4}=\Sigma_{1}^{1}\left(z_{1} \leftrightarrow z_{4}\right)$. Additionally, there is a double logarithmic solution

$$
\begin{aligned}
\Pi\left(\Gamma_{2}\right)= & \varpi\left[\log \left(z_{1}\right) \log \left(z_{2}\right)+\log \left(z_{1}\right) \log \left(z_{3}\right)+\log \left(z_{1}\right) \log \left(z_{4}\right)+\log \left(z_{2}\right) \log \left(z_{3}\right)\right. \\
& \left.+\log \left(z_{2}\right) \log \left(z_{4}\right)+\log \left(z_{3}\right) \log \left(z_{4}\right)\right]+\left(\Sigma_{1}^{2}+\Sigma_{1}^{3}+\Sigma_{1}^{4}\right) \log \left(z_{1}\right) \\
& +\left(\Sigma_{1}^{1}+\Sigma_{1}^{3}+\Sigma_{1}^{4}\right) \log \left(z_{2}\right)+\left(\Sigma_{1}^{1}+\Sigma_{1}^{2}+\Sigma_{1}^{4}\right) \log \left(z_{3}\right) \\
& +\left(\Sigma_{1}^{1}+\Sigma_{1}^{2}+\Sigma_{1}^{3}\right) \log \left(z_{4}\right)+\Sigma_{2}
\end{aligned}
$$

with

$$
\begin{aligned}
\Sigma_{2}= & 4\left(z_{1} z_{2}+z_{3} z_{2}+z_{4} z_{2}+z_{1} z_{3}+z_{1} z_{4}+z_{3} z_{4}\right)+6\left(2 z_{1}^{2} z_{2}+2 z_{1}^{2} z_{3}+2 z_{1}^{2} z_{4}+2 z_{1} z_{2}^{2}\right. \\
& +2 z_{1} z_{3}^{2}+2 z_{1} z_{4}^{2}+11 z_{2} z_{3} z_{1}+11 z_{1} z_{2} z_{4}+11 z_{1} z_{3} z_{4}+2 z_{2} z_{3}^{2} \\
& \left.+2 z_{2} z_{4}^{2}+2 z_{3} z_{4}^{2}+2 z_{2}^{2} z_{3}+2 z_{2}^{2} z_{4}+2 z_{3}^{2} z_{4}+11 z_{2} z_{3} z_{4}\right)+\cdots
\end{aligned}
$$

Together with the holomorphic period (3.39) this completes the period basis.

There is another very compact way of expressing the double logarithmic solution. We define the so called mirror maps

$$
t_{i}=\frac{\Pi\left(\Gamma_{1}^{i}\right)}{2 \pi i \varpi} \quad \text { for } i=1, \ldots, 4 .
$$

Now we can express the double logarithmic solution $\Pi\left(\Gamma_{2}\right)$ in terms of the mirror maps $t_{i}$ for $i=1, \ldots, 4$. For this one has to solve equation (3.44) for the variables $z_{i}$ and plug it into $\Pi\left(\Gamma_{2}\right)$. One obtains

$$
\Pi\left(\Gamma_{2}\right)=\varpi\left(t_{1} t_{2}+t_{1} t_{3}+t_{1} t_{4}+t_{2} t_{3}+t_{2} t_{4}+t_{3} t_{4}\right),
$$

which is so simple since on a K3 surface there are no instanton corrections, see also the discussion in section 3.3.3.

Again after dividing by the inner point and a transformation into the physical parameters (3.35) these six basis solutions can be linearly combined to give the maximal cut integral $\mathcal{F}_{T^{3}}$ at all points in moduli space.

\subsubsection{Extension to inhomogeneous differential operators}

For the full three-loop banana graph we have to extend the differential operator ideal to an inhomogeneous set of operators. We find these inhomogeneities again when we apply the homogeneous system $\mathcal{D}_{1}, \ldots, \mathcal{D}_{4}$ on the geometrical differential $\frac{u \mu_{3}}{P_{3}}$ and perform afterwards an integration over the simplex $\sigma_{3}$. These integrals can only be performed numerically in all four Batyrev coordinates, but fortunately we can guess their exact values. They are ${ }^{22}$

$$
\begin{aligned}
& \mathcal{D}_{1} \Pi_{\sigma_{3}}=0 \\
& \mathcal{D}_{2} \Pi_{\sigma_{3}}=5 \log \left(z_{1}\right)-5 \log \left(z_{2}\right)
\end{aligned}
$$

\footnotetext{
${ }^{22}$ Also here we checked this numerically up to more than 15 digits and for different values of the variables $z_{i}$ for $i=1,2,3$.
} 


$$
\begin{aligned}
& \mathcal{D}_{3} \Pi_{\sigma_{3}}=\log \left(z_{1}\right)+\log \left(z_{2}\right)+\log \left(z_{3}\right)-3 \log \left(z_{4}\right) \\
& \mathcal{D}_{4} \Pi_{\sigma_{3}}=-5 \log \left(z_{3}\right)+5 \log \left(z_{4}\right) .
\end{aligned}
$$

These inhomogeneous differential equations describe all the functions appearing in the Feynman graph (3.31). The missing special solution can be computed with a triple logarithmic ansatz. For example we can take the following function

$$
\begin{aligned}
\varpi_{S}= & -\varpi\left[\log \left(z_{1}\right) \log \left(z_{2}\right) \log \left(z_{3}\right)+\log \left(z_{1}\right) \log \left(z_{3}\right) \log \left(z_{4}\right)\right. \\
& \left.+\log \left(z_{1}\right) \log \left(z_{3}\right) \log \left(z_{4}\right)+\log \left(z_{2}\right) \log \left(z_{3}\right) \log \left(z_{4}\right)\right] \\
& -2\left[\left(z_{1}+z_{2}\right)\left(\log \left(z_{1}\right)+\log \left(z_{2}\right)\right)+\left(z_{1}+z_{3}\right)\left(\log \left(z_{1}\right)+\log \left(z_{3}\right)\right)\right. \\
& +\left(z_{1}+z_{4}\right)\left(\log \left(z_{1}\right)+\log \left(z_{4}\right)\right)+\left(z_{2}+z_{3}\right)\left(\log \left(z_{2}\right)+\log \left(z_{3}\right)\right) \\
& \left.+\left(z_{2}+z_{4}\right)\left(\log \left(z_{2}\right)+\log \left(z_{4}\right)\right)+\left(z_{3}+z_{4}\right)\left(\log \left(z_{2}\right)+\log \left(z_{4}\right)\right)\right] \\
& +2\left[\left(-3 z_{1}+z_{2}+z_{3}+z_{4}\right) \log \left(z_{1}\right)+\left(z_{1}-3 z_{2}+z_{3}+z_{4}\right) \log \left(z_{2}\right)\right. \\
& \left.+\left(z_{1}+z_{2}-3 z_{3}+z_{4}\right) \log \left(z_{3}\right)+\left(z_{1}+z_{2}+z_{3}-3 z_{4}\right) \log \left(z_{4}\right)\right] \\
& +12\left(z_{1}+z_{2}+z_{3}+z_{4}\right)+\cdots .
\end{aligned}
$$

Again, the general solution is then a linear combination of the form $\Pi_{\sigma_{3}}=\varpi_{S}+\lambda_{0} \varpi+$ $\sum_{i=1}^{4} \lambda_{1}^{i} \Pi\left(\Gamma_{1}^{i}\right)+\lambda_{2} \Pi\left(\Gamma_{2}\right)$ with $\lambda_{0}, \lambda_{1}^{i}, \lambda_{2} \in \mathbb{C}$ for $i=1,2,3,4$. We can express $\Pi_{\sigma_{3}}$ through the physical parameters $\left(t, \xi_{1}, \xi_{2}, \xi_{3}, \xi_{4}\right)$ and divide it by the inner point to yield the full three-loop banana Feynman graph (3.31).

\subsubsection{The equal mass case and general properties of the ideal of differential operators}

For the three-loop banana graph not too many results are known in the literature. ${ }^{23}$ In the equal mass case there is an inhomogeneous differential equation

$$
t^{2}(t-4)(t-16) f_{3}^{\prime \prime \prime}(t)+\left(6 t^{3}-90 t^{2}+192 t\right) f_{3}^{\prime \prime}(t)+\left(7 t^{2}-68 t+64\right) f_{3}^{\prime}(t)+(t-4) f_{3}(t)=-4 !
$$

computed in [45]. Restricting our solutions (3.39), (3.40), (3.42) and (3.47) to the equal mass case, dividing by the inner point and transform them to the point at infinity in moduli space they satisfy equation (3.48) showing consistency in this limit.

Let us make some general remarks on the properties of the homogeneous part of the differential operators for periods on K3. We first highlight the structure, which is related to the vanishing string world sheet instantons or unreduced Gromov-Witten invariants on K3 manifolds [48, 49], which is expected to hold more generally for hyperkähler manifolds. This together with (2.35) for $n=2$ and $r=0,1$ implies a structure for the solutions which is reflected also in the classical $W$ invariants of the homogeneous operator $\mathcal{D}_{\mathrm{K} 3}$ in $\mathcal{D}_{\mathrm{K} 3} f(t)=-4$ ! of (3.48) that determines the Feynman graph. To explore the consequences of the vanishing instantons we have to transform the operator for the periods $\int_{\Gamma} \Omega$ with $\Omega$ as in (2.19) to the point of maximal unipotent monodromy, where the instantons are calculated by mirror symmetry in the B-model. That amounts to change the variable from

\footnotetext{
${ }^{23}$ For a discussion on the maximal cut integral in the equal mass case we refere to [47].
} 
$t$ to $z=-1 / u$ by (2.17) and change the dependent function to $f(z)=f_{3}(z) / z$ which yields the operator

$$
\left[\theta^{3}+2 z \theta\left(1+3 \theta+2 \theta^{2}\right)-16 z^{2}\left(6+\theta\left(16+15 \theta+5 \theta^{2}\right)+96 z^{3}\left(6+\theta\left(13+9 \theta+2 \theta^{2}\right)\right)\right] f(z)=0 .\right.
$$

At $z=0$ the unique holomorphic solution is $\varpi=\Pi\left(T^{2}\right)=1+12 z^{2}-48 z^{3}+\mathcal{O}\left(z^{3}\right)$, while the single logarithmic solution starts with $\Pi\left(\Gamma_{1}\right)=\frac{1}{2 \pi i}\left[\varpi \log (z)-2 z+17 z^{2}+\mathcal{O}\left(z^{3}\right)\right]$. The mirror map is defined as $\tau(z)=\Pi\left(\Gamma_{1}\right) / \Pi\left(T^{2}\right)$ and with $q=\exp (2 \pi i \tau)$ one realises that its inverse is

$$
\frac{1}{z(q)}=\frac{1}{q}-2+15 q-32 q^{2}+87 q^{3}-192 q^{4}+343 q^{5}-672 q^{6}+1290 q^{7}+\mathcal{O}\left(q^{8}\right) .
$$

This was identified ${ }^{24}$ as $1 / z(q)=\left(\frac{\eta(\tau) \eta(3 \tau)}{\eta(2 \tau) \eta(6 \tau)}\right)^{6}+4$ the total modular invariant or Hauptmodul of the group $\Gamma_{0}(6)^{+} 3$ [22]. Such identifications have been made for many oneparameter K3 families [50] based on tables for invariants of Hauptmodules for modular groups that features in the monstrous moonshine conjecture [51].

Let $\Pi\left(\Gamma_{2}\right)$ be the double logarithmic solution. Because mirror symmetry maps the period vector $\Pi^{T}=\left(\Pi\left(T^{2}\right), \Pi\left(\Gamma_{1}\right), \Pi\left(\Gamma_{2}\right)\right)$ to the central charges of branes in integer vertical classes $\left(H_{00}, H_{11}^{\text {vert }}, H_{22}\right)$ of the mirror K3, we can calculate $\Sigma^{i j}$ on the mirror and infer that the $n=2$ and $r=0$ relation in $(2.35)$ reads $2 \Pi\left(T^{2}\right) \Pi\left(\Gamma_{2}\right)+m \Pi\left(\Gamma_{1}\right)^{2}=0$, where $m$ is the self intersection of the primitive holomorphic curve spanning $H_{11}^{\text {vert }}(M, \mathbb{Z})$. One finds that the period vector can be written as $\Pi^{T}=\Pi\left(T^{2}\right)\left(1, \tau,-\frac{m}{2} \tau^{2}\right)$. There is also a modular parametrization of $\Pi\left(T^{2}\right)$ namely $z \Pi\left(T^{2}\right)=\frac{(\eta(2 \tau) \eta(6 \tau))^{4}}{(\eta(\tau) \eta(3 \tau))^{2}}$ is the square of periods of a family of elliptic curves associated to $\Gamma_{1}(6)$. The term $\frac{m}{2}$ encodes the classical intersection of the mirror K3 and the absence of $q^{n}$ terms indicates the vanishing of all instanton corrections.

The classical theory see e.g. [52] that goes back to Hermann Schwarz, that was applied already to the one-parameter $\mathrm{K} 3$ in [53], relates the latter fact to the vanishing of the $W_{3}$ invariant of the $\mathrm{K} 3$ operator written generically as

$$
D f=f^{\prime \prime \prime}+3 p(v) f^{\prime \prime}+3 q(v) f^{\prime}+r(v) f=0 .
$$

By a change of the dependent function $g(v)=f(v) \exp \left(\int p d v\right)$ one eliminates the second derivative

$$
g^{\prime \prime \prime}+3 Q(v) g^{\prime}+R(v) g=0
$$

with $R=r-3 p q+2 p^{3}-p^{\prime \prime}$ and $Q=q-p^{2}-p^{\prime}$. Here $Q$ is an invariant of the differential equation, which can be used to introduce a new variable $\tau$, determined as a solution of the Schwarzian equation

$$
\{\tau, v\}=\frac{3}{2} Q
$$

\footnotetext{
${ }^{24}$ Today such identifications of the group and the $\eta$ quotient for a wide class of groups are given by the Webpage of the "On-line Encylopedia of Integer Sequences" at www//oeis.org given enough coefficients of series as in (3.50).
} 
If the second invariant $W_{3}=R-\frac{3}{2} Q^{\prime}=0$ vanishes, the function $h=\frac{d \tau}{d v} g$ satisfies the differential equation ${ }^{25}$

$$
\frac{d^{3}}{d^{3} \tau} h(\tau)=0
$$

with the solution space $\mathbb{C} \oplus \tau \mathbb{C} \oplus \tau^{2} \mathbb{C}$. Schwarz theory determines also the second order linear differential equation

$$
\mathfrak{D} \mathfrak{f}=\mathfrak{f}^{\prime \prime}+2 \mathfrak{q}(v) \mathfrak{f}^{\prime}+\mathfrak{q} \mathfrak{f}(v)=0,
$$

whose ratio of solutions $\tau=\mathfrak{f}_{1} / \mathfrak{f}_{2}$ fulfills (3.53) and which has the property $D=\operatorname{Sym}_{2}(\mathfrak{D})$, which means that the solutions to $D f=0$ are $\mathfrak{f}_{1}^{2}, \mathfrak{f}_{1} \mathfrak{f}_{2}, \mathfrak{f}_{2}^{2}$. It can be found by inverting the following steps: after the trivial observation that $\mathfrak{g}=\mathfrak{f} e^{\int \mathfrak{p} d v}$ fulfills $\mathfrak{g}^{\prime \prime}+\mathfrak{Q} \mathfrak{g}=0$, where $\mathfrak{Q}=\mathfrak{q}-\mathfrak{p}^{2}-\mathfrak{p}^{\prime}$, Schwarz noted that with $\{\tau, v\}=2 \mathfrak{Q}$ defining $\mathfrak{h}=\sqrt{\frac{d \tau}{d v}} \mathfrak{g}$ the function $\mathfrak{h}$ fulfills $\frac{d^{2}}{d \tau^{2}} \mathfrak{h}(\tau)=0$ and hence has solution space $\mathbb{C} \oplus \tau \mathbb{C}$.

If $\mathfrak{Q}=\frac{3}{4} Q$ then the two $\tau(v)$ above are identified. Obviously, the solutions $h$ and $g$ are a symmetric square of the solutions $\mathfrak{h}$ and $\mathfrak{g}$ respectively and one can arrange $\mathfrak{p}$ so that also the solutions $f$ are a symmetric square of the ones of $\mathfrak{f}$. Verrill [22] gives this second order equation for $(3.48)^{26}$ and [46] relates this by changes of the dependent and the independent variable to the differential equation for the equal mass sunset graph (3.30).

Four our solutions of the three-loop banana graph with general masses the analogous structures are the equations (2.36). The first equation together with the vanishing of the genus one worldsheet instantons on K3 [48, 49], implies the simple form in (3.45). The coefficients of the double logarithmic terms are fixed by the intersection theory of the dual curve classes on the mirror K3. The second equation (2.36) becomes more powerful in the multi moduli case and restricts the structure of the solutions as well as the differential ideal in (A.1)-(A.4). One of the strongest hints that automorphic forms also gover the maximal cut graph as solution to (A.1)-(A.4) is the mirror map. The analog of (3.50) given as the multi parameter inversion of (3.40) leads to $1 / z_{i}\left(q_{1}, \ldots, q_{4}\right)$ for $i=1, \ldots, 4$, which have also integer expansions in the $q_{i}=\exp \left(2 \pi i t_{i}\right)$, where $t_{i}=\Pi\left(\Gamma_{1}^{i}\right) /(2 \pi i \varpi)$ are the Kähler parameters of the mirror K3. The natural candidate for these automorphic forms are Borcherds lifts of the type discussed in [54] and applied to lattice polarized K3 as in $[55,56]$. As can be seen from the last two papers the automorphic forms are written naturally in terms of the Kähler parameters $t_{i}$ of the mirror. The relations to the physical parameters are given by the mirror map defined by (3.40) and by (3.35).

Finally, let us comment on the higher loop Banana graphs. For example the analog of the differential operator (3.49) at suitable large volume coordinates derives analogously from the $n=5$ entry of table 1 in [45] as (3.49) from (3.48). It also appears in the Web database explained in $[57,58]$ as AESZ34 and is given by

$$
\begin{aligned}
\mathcal{D}= & \theta^{4}-z\left(35 \theta^{4}+70 \theta^{3}+63 \theta^{2}+28 \theta+5\right)+z^{2}(\theta+1)^{2}\left(259 \theta^{2}+518 \theta+285\right) \\
& -225 z^{3}(\theta+1)^{2}(\theta+2)^{2}
\end{aligned}
$$

\footnotetext{
${ }^{25}$ To prove this one uses the property $\{x, y\}=-\left(\frac{d x}{d y}\right)^{2}\{y, x\}$.

${ }^{26}$ Here $\lambda$ is related to $t$ in (3.48) by $\lambda=t-4$.
} 
One advantage of the solutions at the MUM point is that because of the log structure, in case a factorization of the solutions exist, the analytic solution $\varpi$ must be a pure power of solutions of the lower system. ${ }^{27}$ If one tries to factorize in this way it will not work. The reason can be again understood from (2.35), see [33] for a review. Special geometry implies that the solutions will be $\Pi^{T}=\Pi\left(T^{3}\right)\left(1, \tau, \frac{10}{2} \tau^{2}+\mathcal{O}(q),-\frac{10}{6} \tau^{3}+\mathcal{O}(q)\right)$ and that $\Pi_{3}=-\partial_{t} \Pi_{4}$. The reason that this cannot be a symmetric cube are the genus zero world sheet instantons encoded in the higher series in $q$. For this geometry of the oneparameter family of Barth-Nieto quintics they are not vanishing to all degrees. Subtracting the multi-covering contributions the first $n_{d}^{(0)} \in \mathbb{Z}$ are given for degree $d=1, \ldots, 7$ by $24,48,224,1248,8400,62816,516336$. Despite the integer structures in the $n_{d}^{(0)}$ and the mirror map $1 / z=1 / q+8+28 q+104 q^{2}+654 q^{3}+\mathcal{O}\left(q^{4}\right)$ it will be much more complicated to give closed automorphic expressions for the equal mass four-loop graph then for the general mass three-loop graph.

There are however interesting relations of the periods to modular forms of $\Gamma_{0}(N)$ and algebraic extensions at the rank two attractor points that (3.56) as studied in [59]. At these points the numerator of the Hasse Weil factorises and the exact values of maximal cut integral are given by $L$-function values of holomorphic Hecke Eigenforms forms of weight two and four of $\Gamma_{0}(N)$ [59] or extensions and the quasi-periods of the corresponding meromorphic forms $[60,61]$.

\section{Conclusions and outlook}

The geometric interpretation relating Feynman integrals to Calabi-Yau chain integrals leads to powerful new calculational methods. In particular, the resonant GKZ differential system that was used in the context of mirror symmetry to the period integrals of Calabi-Yau hypersurfaces in toric varities $[7,8,24]$ yields straightforwardly to the maximal cut integral at the point of maximal unipotent monodromy. The advantage of the GKZ differential system is that it uses the symmetries of the Newton polytopes associated to the banana graphs most efficiently. Its disadvantage, namely that it has more solutions and more variables than the actual Calabi-Yau and Feynman integrals, can be overcome using methods from the mirror symmetry application of the GKZ system [7, 8, 24]. The latter allows us to derive the complete homogeneous Picard-Fuchs differential ideal in the physical parameters. The solutions to this differential ideal characterizes the analytic form of the maximal cut integral everywhere in the physical parameter space. The use of the symmetries in this approach turns out to be more efficient than the multi parameter Griffiths reduction method, which gives differential relations between different integrals. Similar differential relations between master integrals for different classes of Feynman graphs appeared in the physics literature in [62-64].

Moreover, at the point of maximal unipotent monodromy we could determine the inhomogeneity by integrating directly the geometrical chain integral after applying the generators of the homogeneous Picard-Fuchs differential ideal to its integrand. The form

\footnotetext{
${ }^{27}$ The easiest way to find the operator (3.55) on a computer might be indeed to take the square root of the unique holomorphic solution $\varpi$ and search for a second order operator that annihilates it.
} 
of the corresponding inhomogeneities turn out to be very simply. This allows us to find an inhomogeneous solution and express for the first time the full mass dependence of the three-loop banana graph analytically. The result is related to the chain integrals that appear in the calculation of open topological string amplitudes.

The GKZ integrals and Feynman integrals can have more general rational functions as integrand than the simple one that is realized for the Banana graph. The scaling invariance that occur in Feynman integrals, lead however typically to GKZ systems related to Calabi-Yau geometries. However, their desingularizations can have much more complicated realizations as the hypersurfaces in toric varieties that feature in this paper. For instance, complete intersections in toric varities or even more exotic cases as Paffian Calabi-Yau spaces in Grassmanians or flag manifolds are conceivable at least in special slices of the moduli space. Nevertheless, we expect that many aspects of the general approach outlined in this paper should apply. In particular, the GKZ system has been applied to the complete intersection three-fold case in [25-27] and to higher dimensional Calabi-Yau manifolds in [28-31]. Recently, progress has been made concerning the more exotic realizations of Calabi-Yau spaces in the $(2,2)$ supersymmetric $2 \mathrm{~d}$ gauge linear $\sigma$ model approach with non-abelian gauge groups. For example in $[32,65]$ the Picard-Fuchs operators for such geometries have been obtained using localization techniques.

Moreover, there are important universal properties that govern the Calabi-Yau periods completely independent of their geometrical realization. In particular, there are the transversality identities (2.29) which have fundamental consequences on the period geometry of Calabi-Yau manifolds, which are very different in even and odd dimensions. Together with some likewise universal properties about the integrality of the mirror map as well as the integrality of instantons and vanishing theorems for the latter, it strongly restricts the classes of automorphic functions that can encode the Feynman integrals.

Our main result is the calculation of the three-loop graph. Let us shortly comment on the possibility to extend our methods to the four loop banana graph: it is possible to find the analogs of (3.3), (3.16) and (3.35) as well as of the differential ideals (3.5), (3.25), and (A.1) - (A.4) for the four-loop graph. Also the inhomogeneous terms (3.7), (3.28) and (3.46) are expected to generalize. With some efforts to code the recursions that follow form the analog of (A.1) - (A.4) as well as (3.47) efficiently, it should be possible to find fast convergent expressions for the four-loop general mass case, just as it is possible for the three-loop case.

\section{Acknowledgments}

We like to thank Mahsa Barzegar, Kilian Bönisch, Ruth Britto, Francis Brown, Philip Candelas, Xenia del la Ossa, Claude Duhr, Mohamed Elmi, Hans Jockers, Rene Klausen, Duco van Straten, Emanuel Scheidegger and Don Zagier for discussion on various aspects of this work as well as very enlightening talks related to the subject. AK wants to thank the LPT-ENS for hospitality and thank Pierre Vanhove for discussions, which have triggered this project. 


\section{A Differential operator ideal of the banana graph}

Here we list a generating set of differential operators which describes the three-loop banana graph in all four physically important Batyrev coordinates.

$$
\begin{aligned}
& \mathcal{D}_{1}=\left(\theta_{1}-\theta_{2}\right)\left(\theta_{3}-\theta_{4}\right) \\
& +z_{1}\left(\theta_{3}-\theta_{4}\right)\left(\theta_{1}-\theta_{2}-\theta_{3}-\theta_{4}\right)+z_{2}\left(\theta_{3}-\theta_{4}\right)\left(\theta_{1}-\theta_{2}+\theta_{3}+\theta_{4}\right) \\
& -2\left(z_{1}-z_{2}\right)\left(z_{3}\left(\theta_{3}+1\right)-z_{4}\left(\theta_{4}+1\right)\right)\left(\theta_{1}+\theta_{2}+\theta_{3}+\theta_{4}+1\right) \\
& \mathcal{D}_{2}=5\left(\theta_{1}-\theta_{2}\right) \theta_{4}-6 \theta_{2}^{2} \\
& +z_{1}\left(2 \theta_{1}^{2}-8 \theta_{1} \theta_{2}+6 \theta_{2}^{2}-6 \theta_{3}^{2}-11 \theta_{4}^{2}+4\left(\theta_{1}+\theta_{2}\right) \theta_{3}+\left(9 \theta_{1}-\theta_{2}-13 \theta_{3}\right) \theta_{4}\right) \\
& +z_{2}\left(17 \theta_{4}^{2}+\left(13 \theta_{1}-9 \theta_{2}+25 \theta_{3}+6\right) \theta_{4}-2\left(\theta_{2}-\theta_{3}\right)\left(4 \theta_{2}+6 \theta_{3}+3\right)\right. \\
& \left.+\theta_{1}\left(8 \theta_{2}+8 \theta_{3}+6\right)\right)+2\left[5 z_{3} z_{4}\left(\theta_{2}-\theta_{1}\right)+z_{1}^{2}\left(\theta_{1}-\theta_{2}-\theta_{3}-\theta_{4}\right)\right. \\
& +z_{2}^{2}\left(\theta_{1}-\theta_{2}+\theta_{3}+\theta_{4}\right)+z_{1} z_{4}\left(3 \theta_{1}+3 \theta_{2}-2 \theta_{3}-8 \theta_{4}-5\right) \\
& +z_{1} z_{3}\left(3\left(\theta_{1}+\theta_{2}-\theta_{3}\right)-2 \theta_{4}\right)+3 z_{1} z_{2}\left(-\theta_{1}+3 \theta_{2}+\theta_{3}+\theta_{4}+2\right) \\
& \left.+z_{2} z_{3}\left(6 \theta_{3}+5 \theta_{4}+6\right)+z_{2} z_{4}\left(5 \theta_{3}+11 \theta_{4}+11\right)\right]\left(\theta_{1}+\theta_{2}+\theta_{3}+\theta_{4}+1\right) \\
& \mathcal{D}_{3}=-3 \theta_{2}^{2}-2 \theta_{2} \theta_{4}+\theta_{1}\left(3 \theta_{2}-2 \theta_{4}\right)+\theta_{4}\left(\theta_{3}+\theta_{4}\right) \\
& -3 z_{1} \theta_{2}\left(-\theta_{1}+\theta_{2}+\theta_{3}\right)-z_{1} \theta_{4}\left(2 \theta_{1}+\theta_{2}-2 \theta_{3}\right)-z_{3} \theta_{4}\left(\theta_{1}+\theta_{2}-\theta_{3}\right)+\left(2 z_{1}-z_{3}\right) \theta_{4}^{2} \\
& -z_{4}\left(\theta_{1}+\theta_{2}+\theta_{3}-\theta_{4}\right)\left(\theta_{4}+1\right)+z_{2}\left(\theta_{1}-\theta_{2}+\theta_{3}+\theta_{4}\right)\left(3 \theta_{2}+8 \theta_{4}+3\right) \\
& +2\left[-2 z_{3} z_{4}\left(\theta_{4}+1\right)+z_{1} z_{4}-3 z_{1} z_{3} \theta_{2}+z_{1}\left(z_{3}+z_{4}\right) \theta_{4}+z_{2} z_{3}\left(3 \theta_{2}+4 \theta_{4}+3\right)\right. \\
& \left.+4 z_{2} z_{4}+4 z_{2}\left(z_{1}+z_{4}\right) \theta_{4}\right]\left(\theta_{1}+\theta_{2}+\theta_{3}+\theta_{4}+1\right) \\
& \mathcal{D}_{4}=-\theta_{2}\left(\theta_{2}+5 \theta_{3}-5 \theta_{4}\right) \\
& +z_{1}\left(2 \theta_{1}^{2}-\left(3 \theta_{2}+\theta_{3}-4 \theta_{4}\right) \theta_{1}+\theta_{2}^{2}-\theta_{3}^{2}-6 \theta_{4}^{2}+4 \theta_{2} \theta_{3}-\left(\theta_{2}+3 \theta_{3}\right) \theta_{4}\right) \\
& +5 z_{4}\left(\theta_{1}-\theta_{2}-\theta_{3}\right)\left(\theta_{1}+\theta_{2}+\theta_{3}-\theta_{4}\right)+5 z_{3} \theta_{4}\left(\theta_{1}+\theta_{2}-\theta_{3}+\theta_{4}\right) \\
& +z_{2}\left[-3 \theta_{2}^{2}+\left(-14 \theta_{3}+11 \theta_{4}-1\right) \theta_{2}+17 \theta_{3}^{2}-8 \theta_{4}^{2}+\theta_{3}\right. \\
& \left.+\theta_{1}\left(3 \theta_{2}+13 \theta_{3}-12 \theta_{4}+1\right)+5 \theta_{3} \theta_{4}+\theta_{4}\right] \\
& +\left[2 z_{1}^{2}\left(\theta_{1}-\theta_{2}-\theta_{3}-\theta_{4}\right)+z_{1} z_{4}\left(11 \theta_{1}-9 \theta_{2}+\theta_{3}-11 \theta_{4}\right)\right. \\
& +z_{1} z_{2}\left(-\theta_{1}+3 \theta_{2}+11 \theta_{3}-9 \theta_{4}+2\right)+z_{1} z_{3}\left(\theta_{1}+11 \theta_{2}-\theta_{3}+\theta_{4}\right) \\
& +2 z_{2} z_{3}\left(-5 \theta_{2}+11 \theta_{3}-5 \theta_{4}+6\right)+2 z_{2} z_{4}\left(5 \theta_{3}-4 \theta_{4}-4\right)+10 z_{3} z_{4}\left(\theta_{4}-\theta_{3}\right) \\
& \left.+2 z_{2}^{2}\left(\theta_{1}-\theta_{2}+\theta_{3}+\theta_{4}\right)\right]\left(\theta_{1}+\theta_{2}+\theta_{3}+\theta_{4}+1\right)
\end{aligned}
$$

Open Access. This article is distributed under the terms of the Creative Commons Attribution License (CC-BY 4.0), which permits any use, distribution and reproduction in any medium, provided the original author(s) and source are credited.

\section{References}

[1] I.M. Gel'fand, A.V. Zelevinskii and M.M. Kapranov, Hypergeometric functions and toric varieties, Funkt. Anal. Prilozhen. 23 (1989) 12.

[2] C. Bogner and S. Weinzierl, Periods and Feynman integrals, J. Math. Phys. 50 (2009) 042302 [arXiv: 0711.4863] [INSPIRE]. 
[3] P.A. Griffiths, Periods of integrals on algebraic manifolds. I. Construction and properties of the modular varieties, Amer. J. Math. 90 (1968) 568.

[4] P.A. Griffiths, Periods of integrals on algebraic manifolds. II. Local study of the period mapping, Amer. J. Math. 90 (1968) 805.

[5] I.M. Gel'fand, A. V. Zelevinskiı̌ and M.M. Kapranov, Newton polyhedra of principal A-determinants, Dokl. Akad. Nauk SSSR 308 (1989) 20.

[6] I.M. Gel'fand, M.M. Kapranov and A.V. Zelevinsky, Generalized Euler integrals and A-hypergeometric functions, Adv. Math. 84 (1990) 255.

[7] S. Hosono, A. Klemm, S. Theisen and S.-T. Yau, Mirror symmetry, mirror map and applications to Calabi-Yau hypersurfaces, Commun. Math. Phys. 167 (1995) 301 [hep-th/9308122] [INSPIRE].

[8] S. Hosono, B.H. Lian and S.-T. Yau, GKZ generalized hypergeometric systems in mirror symmetry of Calabi-Yau hypersurfaces, Commun. Math. Phys. 182 (1996) 535 [alg-geom/9511001] [INSPIRE].

[9] P.A. Griffiths, On the periods of certain rational integrals. I, II, Ann. Math. 90 (1969) 460.

[10] O.V. Tarasov, Connection between Feynman integrals having different values of the space-time dimension, Phys. Rev. D 54 (1996) 6479 [hep-th/9606018] [INSPIRE].

[11] R.N. Lee, Space-time dimensionality D as complex variable: calculating loop integrals using dimensional recurrence relation and analytical properties with respect to D, Nucl. Phys. B 830 (2010) 474 [arXiv:0911.0252] [INSPIRE].

[12] J.L. Bourjaily et al., Traintracks through Calabi-Yau manifolds: scattering amplitudes beyond elliptic polylogarithms, Phys. Rev. Lett. 121 (2018) 071603 [arXiv:1805.09326] [INSPIRE].

[13] J.L. Bourjaily, A.J. McLeod, M. von Hippel and M. Wilhelm, Bounded collection of Feynman integral Calabi-Yau geometries, Phys. Rev. Lett. 122 (2019) 031601 [arXiv:1810.07689] [INSPIRE].

[14] J.L. Bourjaily et al., Embedding Feynman integral (Calabi-Yau) geometries in weighted projective space, JHEP 01 (2020) 078 [arXiv:1910.01534] [INSPIRE].

[15] A. Primo and L. Tancredi, On the maximal cut of Feynman integrals and the solution of their differential equations, Nucl. Phys. B 916 (2017) 94 [arXiv:1610.08397] [INSPIRE].

[16] L. de la Cruz, Feynman integrals as A-hypergeometric functions, JHEP 12 (2019) 123 [arXiv: 1907.00507] [INSPIRE].

[17] R.P. Klausen, Hypergeometric series representations of Feynman integrals by GKZ hypergeometric systems, arXiv: 1910.08651 [INSPIRE].

[18] S. Bloch, M. Kerr and P. Vanhove, Local mirror symmetry and the sunset Feynman integral, Adv. Theor. Math. Phys. 21 (2017) 1373.

[19] R. Bonciani et al., Evaluating a family of two-loop non-planar master integrals for Higgs + jet production with full heavy-quark mass dependence, JHEP 01 (2020) 132 [arXiv: 1907.13156] [INSPIRE].

[20] M.-X. Huang, A. Klemm and M. Poretschkin, Refined stable pair invariants for E-, M- and [p,q]-strings, JHEP 11 (2013) 112 [arXiv:1308.0619] [INSPIRE]. 
[21] S. Abreu, M. Becchetti, C. Duhr and R. Marzucca, Three-loop contributions to the $\rho$ parameter and iterated integrals of modular forms, JHEP 02 (2020) 050 [arXiv:1912.02747] [INSPIRE].

[22] H.A. Verrill, Root lattices and pencils of varieties, J. Math. Kyoto Univ. 36 (1996) 423.

[23] P. Vanhove, The physics and the mixed Hodge structure of Feynman integrals, Proc. Symp. Pure Math. 88 (2014) 161 [arXiv:1401.6438] [INSPIRE].

[24] V.V. Batyrev, Dual polyhedra and mirror symmetry for Calabi-Yau hypersurfaces in toric varieties, J. Alg. Geom. 3 (1994) 493.

[25] S. Hosono, A. Klemm, S. Theisen and S.-T. Yau, Mirror symmetry, mirror map and applications to complete intersection Calabi-Yau spaces, Nucl. Phys. B 433 (1995) 501 [hep-th/9406055] [INSPIRE].

[26] V.V. Batyrev and D. van Straten, Generalized hypergeometric functions and rational curves on Calabi-Yau complete intersections in toric varieties, Comm. Math. Phys. 168 (1995) 493.

[27] V.V. Batyrev and L.A. Borisov, On Calabi-Yau complete intersections in toric varieties, in Higher-dimensional complex varieties, M. Andreatta et al. eds., de Gruyter, Berlin (1996).

[28] B.R. Greene, D.R. Morrison and M.R. Plesser, Mirror manifolds in higher dimension, Commun. Math. Phys. 173 (1995) 559 [hep-th/9402119] [INSPIRE].

[29] P. Mayr, Mirror symmetry, $N=1$ superpotentials and tensionless strings on Calabi-Yau four folds, Nucl. Phys. B 494 (1997) 489 [hep-th/9610162] [INSPIRE].

[30] A. Klemm, B. Lian, S.S. Roan and S.-T. Yau, Calabi-Yau fourfolds for M-theory and F-theory compactifications, Nucl. Phys. B 518 (1998) 515 [hep-th/9701023] [INSPIRE].

[31] N. Cabo Bizet, A. Klemm and D. Vieira Lopes, Landscaping with fluxes and the E8 Yukawa Point in F-theory, arXiv:1404.7645 [INSPIRE].

[32] A. Gerhardus and H. Jockers, Quantum periods of Calabi-Yau fourfolds, Nucl. Phys. B 913 (2016) 425 [arXiv : 1604.05325] [INSPIRE].

[33] A. Klemm, The B-model approach to topological string theory on Calabi-Yau n-folds, in B-model Gromov-Witten theory, E. Clader and Y.B. Ruan eds., Springer, Germany (2018).

[34] T.-F. Feng, C.-H. Chang, J.-B. Chen and H.-B. Zhang, GKZ-hypergeometric systems for Feynman integrals, Nucl. Phys. B 953 (2020) 114952 [arXiv:1912.01726] [INSPIRE].

[35] C. Bogner and S. Weinzierl, Feynman graph polynomials, Int. J. Mod. Phys. A 25 (2010) 2585 [arXiv: 1002 . 3458] [INSPIRE].

[36] R. Bott and L.W. Tu, Differential forms in algebraic topology, Graduate Texts in Mathematics volume 82, Springer, Germany (1982).

[37] D.A. Cox, J.B. Little and H.K. Schenck, Toric varieties, Graduate Studies in Mathematics volume 124, American Mathematical Society, Providence, U.S.A. (2011).

[38] http://doc.sagemath.org/html/en/reference/schemes/sage/schemes/toric/variety.html

[39] S. Bloch, M. Kerr and P. Vanhove, Local mirror symmetry and the sunset Feynman integral, Adv. Theor. Math. Phys. 21 (2017) 1373 [arXiv:1601.08181] [InSPIRE].

[40] V.V. Batyrev and D. van Straten, Generalized hypergeometric functions and rational curves on Calabi-Yau complete intersections in toric varieties, Commun. Math. Phys. 168 (1995) 493 [alg-geom/9307010] [INSPIRE]. 
[41] J.M. Henn, Lectures on differential equations for Feynman integrals, J. Phys. A 48 (2015) 153001 [arXiv: 1412.2296] [INSPIRE].

[42] R.L. Bryant and P.A. Griffiths, Some observations on the infinitesimal period relations for regular threefolds with trivial canonical bundle, in Arithmetic and geometry. Volume II, Y. Tschinkel and Y.G. Zarhin eds., Birkhäuser, Boston U.S.A. (1983).

[43] S. Li, B.H. Lian and S.-T. Yau, Picard-Fuchs equations for relative periods and Abel-Jacobi map for Calabi-Yau hypersurfaces, arXiv:0910.4215 [INSPIRE].

[44] C. Itzykson and J.B. Zuber, Quantum field theory, International Series In Pure and Applied Physics, McGraw-Hill, New York U.S.A. (1980).

[45] P. Vanhove, The physics and the mixed Hodge structure of Feynman integrals, Proc. Symp. Pure Math. 88 (2014) 161 [arXiv:1401.6438] [InSPIRE].

[46] J. Broedel et al., An analytic solution for the equal-mass banana graph, JHEP 09 (2019) 112 [arXiv: 1907.03787] [INSPIRE].

[47] A. Primo and L. Tancredi, Maximal cuts and differential equations for Feynman integrals. An application to the three-loop massive banana graph, Nucl. Phys. B 921 (2017) 316 [arXiv: 1704.05465] [INSPIRE].

[48] D. Maulik, R. Pandharipande and R.P. Thomas, Curves on K3 surfaces and modular forms, J. Topol. 3 (2010) 937.

[49] G. Oberdieck and R. Pandharipande, Curve counting on $K 3 \times E$, the Igusa cusp form $\chi_{10}$, and descendent integration, in K3 surfaces and their moduli, C. Faber et al. eds., Springer, Germany (2016).

[50] B.H. Lian and S.-T. Yau, Mirror maps, modular relations and hypergeometric series 1, hep-th/9507151 [INSPIRE].

[51] J.H. Conway and S.P. Norton, Monstrous moonshine, Bull. London Math. Soc. 11 (1979) 308.

[52] A.R. Forsyth, Theory of differential equations. 1. Exact equations and Pfaff's problem; 2, 3. Ordinary equations, not linear; 4. Ordinary linear equations; 5, 6. Partial differential equations, Dover Publications Inc., New York U.S.A. (1959).

[53] W. Lerche, D.J. Smit and N.P. Warner, Differential equations for periods and flat coordinates in two-dimensional topological matter theories, Nucl. Phys. B 372 (1992) 87 [hep-th/9108013] [INSPIRE].

[54] R.E. Borcherds, Automorphic forms with singularities on Grassmannians, Invent. Math. 132 (1998) 491.

[55] A. Klemm and M. Mariño, Counting BPS states on the enriques Calabi-Yau, Commun. Math. Phys. 280 (2008) 27 [hep-th/0512227] [INSPIRE].

[56] T.W. Grimm, A. Klemm, M. Mariño and M. Weiss, Direct integration of the topological string, JHEP 08 (2007) 058 [hep-th/0702187] [INSPIRE].

[57] G. Almkvist, C. van Enckevort, D. van Straten and W. Zudilin, Tables of Calabi-Yau equations, math.AG/0507430.

[58] D. van Straten, Calabi-Yau operators, in Uniformization, Riemann-Hilbert correspondence, Calabi-Yau manifolds \& Picard-Fuchs equations, L. Ji and S.T. Yau eds., International Press, Somerville U.S.A. (2018). 
[59] P. Candelas, X. de la Ossa, M. Elmi and D. van Straten, A one parameter family of calabi-yau manifolds with attractor points of rank two, to appear.

[60] A. Klemm, E. Scheidegger and D. Zagier, Periods and quasiperiods of modular forms and $d$-brane masses for the mirror quintic, to appear.

[61] K. Boenisch et al., to appear.

[62] H. Frellesvig and C.G. Papadopoulos, Cuts of Feynman integrals in Baikov representation, JHEP 04 (2017) 083 [arXiv: 1701.07356] [INSPIRE].

[63] J. Bosma, M. Sogaard and Y. Zhang, Maximal cuts in arbitrary dimension, JHEP 08 (2017) 051 [arXiv: 1704.04255] [INSPIRE].

[64] M. Harley, F. Moriello and R.M. Schabinger, Baikov-Lee representations of cut Feynman integrals, JHEP 06 (2017) 049 [arXiv:1705.03478] [INSPIRE].

[65] A. Gerhardus, H. Jockers and U. Ninad, The geometry of gauged linear $\sigma$-model correlation functions, Nucl. Phys. B 933 (2018) 65 [arXiv:1803.10253] [INSPIRE]. 